\title{
Blood bacterial resistant investigation collaborative system (BRICS) report: a national surveillance in China from 2014 to 2019
}

\author{
Yunbo Chen 1,2, Jinru Ji ${ }^{1,2}$, Chaoqun Ying 1,2, Zhiying Liu ${ }^{1,2}$, Qing Yang ${ }^{1,2}$, Haishen Kong 1,2, \\ Yonghong Xiao ${ }^{1,2^{*}}$ (D) and Blood Bacterial Resistant Investigation Collaborative System (BRICS) Study Group
}

\begin{abstract}
Background: In this first national bloodstream infection (BSI) surveillance program in China, we assessed the composition of pathogenic bacteria and the trends for antimicrobial susceptibility over a 6-year period in China.

Methods: Blood bacterial isolates from patients at hospitals participating in the Blood Bacterial Resistant Investigation Collaborative System (BRICS) were collected from January 2014 to December 2019. Only the first isolate of a species per patient was eligible over the full study period. Antibiotic-susceptibility testing was conducted by agar-dilution or broth-dilution methods as recommended by the Clinical and Laboratory Standards Institute (CLSI). WHONET 5.6 was used to analyze data.
\end{abstract}

Results: During the study period, 27,899 bacterial strains were collected. Gram-positive organisms accounted for 29.5\% (8244) of the species identified and Gram-negative organisms accounted for $70.5 \%(19,655)$. The mostcommonly isolated organisms in blood cultures were Escherichia coli, Klebsiella pneumoniae, Staphylococcus aureus, coagulase-negative Staphylococci, and Acinetobacter baumannii. The prevalence of multidrug-resistant organisms, such as E. coli, K. pneumoniae, A. baumannii was higher in tertiary hospitals, whereas extended-spectrum, $\beta$-lactamaseproducing E. coli (ESBL-E. coli), carbapenem-resistant A. baumannii were more prevalent in economically-developing areas. The prevalence of methicillin-resistant S. aureus declined from 39.0\% (73/187) in 2014 to $25.9 \%(230 / 889)$ in $2019(p<0.05)$. The prevalence of ESBL-E. coli dropped from 61.2\% $(412 / 673)$ to $51.0 \%(1878 / 3,683)$ over time $(p<0.05)$, and carbapenem-resistant $E$. coli remained low prevalence $(<2 \% ; 145 / 9944 ; p=0.397)$. In contrast, carbapenem-resistant K. pneumoniae increased markedly from 7.0\% (16/229) in 2014 to 19.6\% $(325 / 1,655)$ in $2019(p<0.05)$.

Conclusion: E. coli and K. pneumoniae were the leading causes of BSI during the 6-year study period. The major resistant pathogens declined or remained stable, whereas carbapenem-resistant $K$. pneumoniae continued to increase, which poses a great therapeutic challenge for BSIs.

Keywords: Resistance, Bacterial, Bloodstream infection, National resistance surveillance

\footnotetext{
*Correspondence: xiao-yonghong@163.com

'State Key Laboratory for Diagnosis and Treatment of Infectious Diseases,

The First Affiliated Hospital, Zhejiang University School of Medicine, Hangzhou 310003, China

Full list of author information is available at the end of the article
}

\section{Background}

Antimicrobial resistance (AMR) has become a serious public health threat across the world. AMR control is a priority for the World Health Organization (WHO) [1]. According to WHO recommendations, AMR surveillance is an important part of the AMR control strategy. Furthermore, the data regarding microbiological original author(s) and the source, provide a link to the Creative Commons licence, and indicate if changes were made. The images or other third party material in this article are included in the article's Creative Commons licence, unless indicated otherwise in a credit line to the material. If material is not included in the article's Creative Commons licence and your intended use is not permitted by statutory regulation or exceeds the permitted use, you will need to obtain permission directly from the copyright holder. To view a copy of this licence, visit http://creativecommons.org/licenses/by/4.0/. The Creative Commons Public Domain Dedication waiver (http://creativeco mmons.org/publicdomain/zero/1.0/) applies to the data made available in this article, unless otherwise stated in a credit line to the data. 
composition and AMR profiles will guide antimicrobial prescriptions [2]. However, almost all bacterial resistance surveillances programs in China are laboratory-based surveillance strategies with potential biases resulting from optional sample collection. Therefore, it is imperative to carry out infection-defined surveillance to overcome this limitation.

Bloodstream infection (BSI) is a growing public health concern worldwide, with high mortality [3, 4]. It was estimated that the BSI incidence ranged between 113 and 204 per 100,000 in the population [5]. Inappropriate antibiotic therapy for BSI was independently associated with increased risk of mortality [6]. Microbiological epidemiology and bacterial resistance data on BSI will provide a reference for the best empirical antimicrobial therapy [7]. In the face of increasing AMR, precise surveillance has become important in defining the species distribution and resistance of pathogens causing BSI, and thus provide the basis for appropriate empirical therapy.

To comprehensively understand and accurately analyze the microbiological epidemiology and resistance profiles of BSI in China, we initiated the Blood Bacterial Resistant Investigation Collaborative System (BRICS) program in 2014. As an infection-defined surveillance initiative, information on the pathogen distribution and AMR of BSI bacteria was collected from participating hospitals covering 18 provinces in mainland China.

\section{Material and methods}

\section{Study period and setting}

A total of 52 hospitals (100,712 beds), which included 23 tertiary hospitals and 29 non-tertiary hospitals covering 18 provinces in mainland China during 2014-2019, participated in the BRICS program. All participating hospitals were equipped with a qualified microbiology laboratory and followed standardized operational programs. All laboratories participated in one external quality control program at least yearly by either the National Center for Clinical Laboratories or the local province center for clinical laboratories.

\section{Bacterial isolate collection}

Only strains isolated from blood were collected. Blood culture results of patients with only skin contaminants were considered contamination, and patients with both BSI and contamination were classified as having BSI [8]. Coagulase-negative Staphylococci, Bacillus species, viridans group Streptococci, Corynebacterium species, Propionibacterium species, Aerococcus species, and Micrococcus species from a single positive culture were excluded as contaminants, while all Brucella species were excluded due to the Biosafety Law of China. Only the first isolate of a species per patient was eligible over the full study period. All participating hospitals transferred their strains to the central laboratory quarterly. The central laboratory confirmed the identity of the isolates received using matrix-assisted laser desorption/ionization time of flight mass spectrometry (Bruker Diagnostics, Bremen, Germany) and stored the strains in Microbank ${ }^{\circledR}$ tubes at $-80^{\circ} \mathrm{C}$.

\section{Antimicrobial susceptibility testing}

The antibiotic susceptibilities (minimum inhibitory concentration, MIC) of clinical isolates were determined by agar-dilution or broth-dilution methods [9] at the central laboratory. Extended-spectrum $\beta$-lactamase (ESBL) production in Escherichia coli, Klebsiella pneumonia, and Proteus mirabilis using disk diffusion, and inducible clindamycin resistance in Staphylococcus species, Streptococcus pneumonia, and Streptococcus spp. $\beta$-hemolytic group were determined. $\beta$-hemolytic group determination using disk diffusion (D-zone test) was performed according to the Clinical and Laboratory Standards Institute (CLSI) [9]. The results of MICs were interpreted according to CLSI criteria or European Committee on Antimicrobial Susceptibility Testing (EUCAST) (https:// www.eucast.org). Staphylococcus aureus ATCC 29,213, Enterococcus faecalis ATCC 29,212, S. pneumonia ATCC 700,603, E. coli ATCC 25,922, K. pneumoniae ATCC 27,853, and Pseudomonas aeruginosa ATCC 27,853 were included as quality controls.

Polymicrobial bacteremia is defined as a bacteremic episode due to at least two different organisms isolated from the same blood sample, while monomicrobial bacteremia is defined as only one organism isolated in the blood sample [10]. Contaminants are defined as a growth of bacteria in the blood culture bottle that were not present in the patient's bloodstream but were introduced during sample collection [11]. For surveillance purpose, carbapenem-resistant Enterobacteriaeceae was defined as demonstrating resistance based upon antimicrobial susceptibility test results to at least one of the following carbapenems: ertapenem, meropenem, or imipenem according to the Centers for Disease Control and Prevention (CDC) of USA [12]. Multidrug-resistant (MDR) is defined as non-susceptibility to at least one agent in three or more antimicrobial categories and pan-drug-resistant (PDR) is defined as resistance to all antibiotic classes available for empirical treatment [13].

\section{Data and statistical analysis}

To compare resistance profiles in different economic areas, a developed or developing area was defined as having a per capita gross domestic product $($ GDP) $\geq 11,000$ USD or $<11,000$ USD in 2019. WHONET 5.6 was used to analyze the distribution and resistance rates of the 
isolates. To assess trends in proportions of resistant isolates over time, the Cochran-Armitage $\chi^{2}$ test was used. The Chi-square test was used to compare rates for different hospital levels and regions. Significance was assumed at a $p$-value $<0.05$. Statistical analysis was performed using SPPS (version 9).

\section{Ethics requirements}

For this observational study, the need for patient consent was not required. Data were not identifiable back to the patients from whom they originated; an ethics approval was waived.

\section{Results}

\section{Bacterial isolate collection}

A total of 52 hospitals from 2014 to 2019 participated in BRICS. There were 8 tertiary hospitals and 13 non-tertiary hospitals that participated over the entire duration of the surveillance program, while other hospitals participated for 1 to 5 years. The number of participating hospitals per year were as follows: 21 in 2014, 26 in 2015, 27 in 2016, 30 in 2017, 39 in 2018, and 49 in 2019.

Over the 6-year period, 27,899 isolates were collected in total; Gram-positive organisms (GPO) accounted for $29.5 \%(8244 / 27,899)$ and Gram-negative organisms (GNO) for $70.5 \%(19,655 / 27,899)$. GNO increased from $58.4 \%$ in 2014 to $73.0 \%$ in 2019 . E. coli and K. pneumoniae were the most common organisms overall, followed by $S$. aureus, coagulase-negative Staphylococci
(CoNS), Acinetobacter baumannii, and $P$. aeruginosa (Fig. 1). Notably, E. coli increased $29.1 \%$ to $38.5 \%$ during the study period. A higher proportion of CoNS were detected in tertiary hospitals and developing areas $(10.1 \%$ and $12.1 \%$, respectively) than those in non-tertiary hospitals or developed areas ( $9.4 \%$ and $7.7 \%$, respectively). The percentage of $E$. coli was higher in non-tertiary hospitals (38.8\%) than in tertiary hospitals (32.5\%). P. aeruginosa and $A$. baumannii were more often detected in tertiary hospitals (Additional file 1: Table S1).

\section{Bacterial resistance and trends over time Resistance in Gram-positive organisms}

The overall prevalence of methicillin-resistant Staphylococcus aureus (MRSA) declined during the study period from $39.0 \%$ to $25.9 \%(p<0.05)$ (Table 1, Fig. 2$)$. No vancomycin resistance, or "MIC creep" was detected over time. Of note, the prevalence of MRSA was higher in developed regions (33.9\% vs $28.3 \%$ in developing regions), but no differences across different hospital levels was observed (Additional file 1: Table S2). Moreover, we observed a decline in the proportion of rifampicin-resistant $S$. aureus during the study period (5.3\% in 2014 and $1.3 \%$ in $2019, p<0.05)$.

The methicillin-resistant, coagulase-negative Staphylococci (MRCNS) prevalence was $74.3 \%$ and no glycopeptide-resistant MRCNS were detected (Additional file 1: Table S2). Compared with MRSA, MRCNS exhibited higher resistance rates to fluoroquinolones

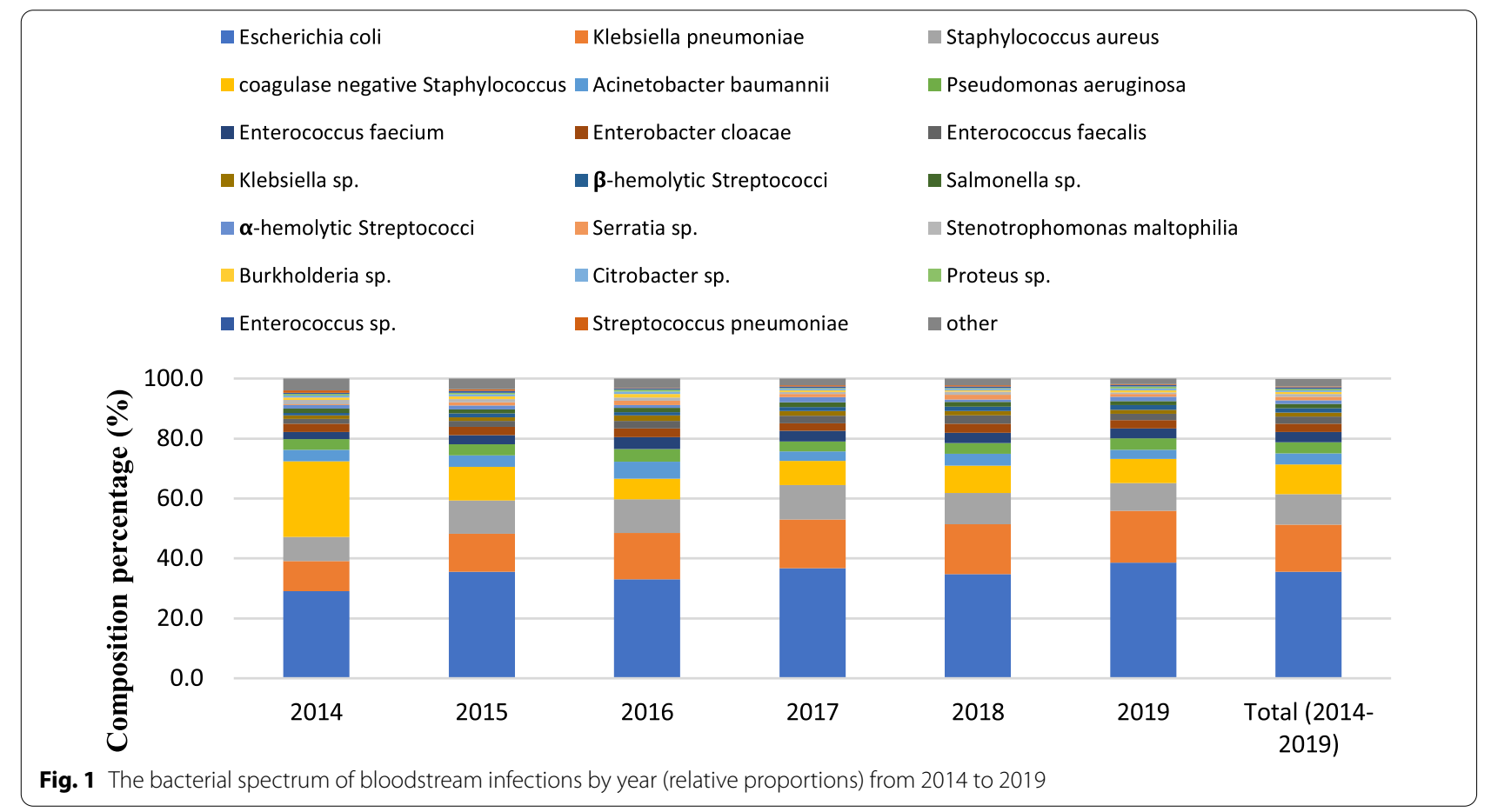


Table 1 The susceptibility and resistance trends of Gram-positive pathogens to antimicrobial agents

\begin{tabular}{|c|c|c|c|c|c|c|c|c|c|c|c|}
\hline & \multicolumn{5}{|c|}{ All strains (204-2019) } & \multicolumn{2}{|c|}{2014} & \multicolumn{2}{|l|}{2015} & \multicolumn{2}{|l|}{2016} \\
\hline & Number & $\mathrm{MIC}_{50}$ & $\mathrm{MIC}_{90}$ & $\% \mathrm{R}$ & $\% \mathrm{~S}$ & $\% R$ & $\% \mathrm{~S}$ & $\% R$ & $\% \mathrm{~S}$ & $\% R$ & $\% \mathrm{~S}$ \\
\hline \multicolumn{12}{|l|}{ S. aureus } \\
\hline MDR & 2843 & - & - & 54.9 & - & 55.6 & - & 55.1 & - & 60.9 & - \\
\hline penicillin G & 2667 & 0.5 & 16 & 87.9 & 12.1 & 91.2 & 8.8 & 88.1 & 11.9 & 84.2 & 15.8 \\
\hline oxacillin & 2843 & 0.5 & 32 & 31.3 & 68.7 & 39.0 & 61.0 & 34.8 & 65.2 & 35.9 & 64.1 \\
\hline amikacin & 2843 & 4 & 8 & 3.7 & 91.6 & 3.2 & 86.6 & 5.1 & 90.2 & 4.9 & 92.1 \\
\hline gentamicin & 2360 & 0.5 & 32 & 15.3 & 84.1 & - & - & - & - & 17.4 & 81.7 \\
\hline rifampicin & 2843 & 0.016 & 0.016 & 3.0 & 96.6 & 5.3 & 94.1 & 3.0 & 95.9 & 4.1 & 95.3 \\
\hline ciprofloxacin & 2360 & 0.5 & 16 & 21.3 & 74.6 & - & - & - & - & 23.9 & 71.4 \\
\hline levofloxacin & 2843 & 0.25 & 16 & 17.9 & 80.7 & 21.4 & 76.5 & 19.6 & 78.7 & 16.2 & 81.3 \\
\hline moxifloxacin & 2843 & 0.125 & 4 & 17.6 & 78.8 & 23.5 & 68.4 & 20.3 & 62.8 & 17.9 & 78.5 \\
\hline $\begin{array}{l}\text { trimethoprim/sul- } \\
\text { famethoxazole }\end{array}$ & 2843 & 0.064 & 0.25 & 2.8 & 97.2 & 4.8 & 95.2 & 7.1 & 92.9 & 3.9 & 96.1 \\
\hline clindamycin & 2843 & 0.25 & 32 & 37.0 & 60.4 & 29.9 & 67.4 & 35.8 & 61.1 & 33.1 & 60.4 \\
\hline erythromycin & 2843 & 32 & 32 & 60.1 & 31.5 & 64.7 & 33.7 & 65.5 & 28.0 & 63.3 & 27.4 \\
\hline daptomycin & 2843 & 0.5 & 1 & 0 & 98.9 & 0 & 97.9 & 0 & 98.3 & 0 & 97.2 \\
\hline linezolid & 2843 & 1 & 2 & 0 & 100 & 0 & 100 & 0 & 100 & 0 & 100 \\
\hline vancomycin & 2843 & 1 & 2 & 0 & 100 & 0 & 100 & 0 & 100 & 0 & 100 \\
\hline teicoplanin & 2360 & 0.5 & 2 & 0 & 100 & - & - & - & - & 0 & 100 \\
\hline tetracycline & 2843 & 0.25 & 32 & 19.4 & 77.2 & 23.5 & 72.7 & 24.0 & 75.7 & 25.0 & 70.6 \\
\hline tigecycline & 2843 & 0.125 & 0.25 & 1.2 & 98.8 & 1.1 & 98.9 & 0.7 & 99.3 & 4.3 & 95.7 \\
\hline \multicolumn{12}{|l|}{ E. faecium } \\
\hline MDR & 950 & - & - & 92.7 & - & 85.7 & - & 81.0 & - & 91.2 & - \\
\hline penicillin G & 950 & 32 & 64 & 88.8 & 11.2 & 87.5 & 12.5 & 87.3 & 12.7 & 86.7 & 13.3 \\
\hline ampicillin & 950 & 32 & 32 & 86.8 & 13.2 & 82.1 & 17.9 & 88.6 & 11.4 & 83.4 & 16.6 \\
\hline rifampicin & 815 & 8 & 8 & 82.2 & 11.3 & - & - & - & - & 74.6 & 15.5 \\
\hline ciprofloxacin & 815 & 16 & 32 & 91.9 & 6.0 & - & - & - & - & 86.7 & 8.8 \\
\hline levofloxacin & 950 & 16 & 32 & 86.3 & 8.6 & 96.4 & 1.8 & 77.2 & 13.9 & 84.0 & 11.6 \\
\hline erythromycin & 950 & 32 & 32 & 87.9 & 3.9 & 83.9 & 1.8 & 81.0 & 6.3 & 87.8 & 5.0 \\
\hline daptomycin & 950 & 2 & 4 & 0.1 & 99.9 & 0 & 100 & 1.3 & 98.7 & 0 & 100 \\
\hline linezolid & 950 & 2 & 2 & 0.5 & 93.2 & 0 & 100 & 1.3 & 98.7 & 0 & 100 \\
\hline vancomycin & 949 & 1 & 2 & 0.5 & 97.9 & 0 & 100 & 1.3 & 98.7 & 0 & 100 \\
\hline teicoplanin & 815 & 0.25 & 1 & 0.2 & 99.8 & - & - & - & - & 0 & 100 \\
\hline tigecycline & 950 & 0.064 & 0.5 & 0.3 & 87.4 & 0 & 98.2 & 0 & 96.2 & 1.1 & 98.9 \\
\hline \multicolumn{12}{|l|}{ E. faecalis } \\
\hline MDR & 636 & - & - & 39.2 & - & 40.0 & - & 19.2 & - & 33.6 & - \\
\hline Penicillin G & 636 & 2 & 8 & 9.0 & 91.0 & 17.1 & 82.9 & 9.6 & 90.4 & 9.7 & 90.3 \\
\hline Ampicillin & 636 & 1 & 4 & 7.4 & 92.6 & 11.4 & 88.6 & 0 & 100 & 10.6 & 89.4 \\
\hline Rifampicin & 549 & 4 & 8 & 50.1 & 23.5 & - & - & - & - & 52.2 & 11.5 \\
\hline Ciprofloxacin & 549 & 1 & 16 & 25.7 & 57.2 & - & - & - & - & 21.2 & 64.6 \\
\hline Levofloxacin & 636 & 2 & 16 & 26.1 & 64.5 & 37.1 & 60.0 & 44.2 & 55.8 & 18.6 & 76.1 \\
\hline Erythromycin & 636 & 32 & 32 & 58.3 & 13.5 & 68.6 & 100 & 51.9 & 98.1 & 61.1 & 18.6 \\
\hline Daptomycin & 636 & 1 & 2 & 0.3 & 98.4 & 0 & 95.3 & 1.9 & 98.1 & 0.9 & 97.3 \\
\hline Linezolid & 636 & 2 & 2 & 1.3 & 98.7 & 0 & 100 & 3.8 & 96.2 & 0.9 & 99.1 \\
\hline Vancomycin & 636 & 1 & 2 & 0 & 99.4 & 0 & 100 & 0 & 100 & 0 & 100 \\
\hline Teicoplanin & 549 & 0.25 & 0.25 & 0.2 & 99.8 & - & - & - & - & 0 & 100 \\
\hline \multirow[t]{3}{*}{ Tigecycline } & 636 & 0.125 & 0.25 & 0.6 & 95.4 & 0 & 97.1 & 0 & 65.4 & 0 & 100 \\
\hline & \multicolumn{2}{|c|}{2017} & & \multicolumn{2}{|l|}{2018} & \multicolumn{3}{|c|}{2019} & \multirow{2}{*}{\multicolumn{2}{|c|}{ Trend }} & \multirow[t]{2}{*}{$p$} \\
\hline & $\% R$ & $\% \mathrm{~S}$ & & $\% R$ & $\% \mathrm{~S}$ & & & $\% \mathrm{~S}$ & & & \\
\hline \multicolumn{12}{|l|}{ S. aureus } \\
\hline MDR & 54.2 & - & & 48.3 & - & & & - & - & & 0.590 \\
\hline
\end{tabular}


Table 1 (continued)

\begin{tabular}{|c|c|c|c|c|c|c|c|c|}
\hline & \multicolumn{2}{|l|}{2017} & \multicolumn{2}{|l|}{2018} & \multicolumn{2}{|l|}{2019} & \multirow[t]{2}{*}{ Trend } & \multirow[t]{2}{*}{$p$} \\
\hline & $\% R$ & $\% \mathrm{~S}$ & $\% R$ & $\% \mathrm{~S}$ & $\% R$ & $\% \mathrm{~S}$ & & \\
\hline penicillin G & 81.2 & 18.8 & 91.1 & 8.9 & 90.7 & 9.3 & $\downarrow$ & 0.004 \\
\hline oxacillin & 33.5 & 66.5 & 29.9 & 70.1 & 25.9 & 74.1 & $\downarrow$ & 0.000 \\
\hline amikacin & 6.0 & 84.3 & 2.4 & 92.3 & 2.2 & 95.7 & $\downarrow$ & 0.006 \\
\hline gentamicin & 19.5 & 80.5 & 15.1 & 84.0 & 12.4 & 87.2 & $\downarrow$ & 0.002 \\
\hline rifampicin & 6.0 & 94.0 & 1.5 & 98.2 & 1.3 & 98.2 & $\downarrow$ & 0.000 \\
\hline ciprofloxacin & 22.2 & 72.3 & 21.9 & 77.0 & 19.0 & 75.9 & - & 0.031 \\
\hline levofloxacin & 20.2 & 79.3 & 21.3 & 76.3 & 14.4 & 85.3 & $\downarrow$ & 0.050 \\
\hline moxifloxacin & 20.2 & 79.3 & 18.0 & 78.9 & 13.7 & 86.1 & $\downarrow$ & 0.000 \\
\hline $\begin{array}{l}\text { trimethoprim/sulfameth- } \\
\text { oxazole }\end{array}$ & 1.0 & 99.0 & 3.5 & 96.5 & 0.9 & 99.1 & $\downarrow$ & 0.000 \\
\hline clindamycin & 33.7 & 62.7 & 29.1 & 69.4 & 47.4 & 52.0 & $\uparrow$ & 0.000 \\
\hline erythromycin & 62.2 & 22.7 & 59.4 & 32.2 & 55.0 & 38.2 & $\downarrow$ & 0.000 \\
\hline daptomycin & 0 & 98.3 & 0 & 100 & 0 & 100 & - & NA \\
\hline linezolid & 0 & 100 & 0 & 100 & 0 & 100 & - & 0.021 \\
\hline vancomycin & 0 & 100 & 0 & 100 & 0 & 100 & - & NA \\
\hline teicoplanin & 0 & 100 & 0 & 100 & 0 & 100 & - & NA \\
\hline tetracycline & 23.1 & 73.7 & 14.0 & 79.2 & 15.4 & 82.9 & $\downarrow$ & 0.000 \\
\hline tigecycline & 1.2 & 98.8 & 0.4 & 99.6 & 0.1 & 99.9 & $\downarrow$ & 0.000 \\
\hline \multicolumn{9}{|l|}{ E. faecium } \\
\hline MDR & 95.4 & - & 98.3 & - & 93.5 & - & $\uparrow$ & 0.000 \\
\hline penicillin G & 90.8 & 9.2 & 89.4 & 10.6 & 89.5 & 10.5 & - & 0.364 \\
\hline ampicillin & 90.0 & 10.0 & 87.7 & 12.3 & 87.4 & 12.6 & - & 0.320 \\
\hline rifampicin & 77.7 & 10.8 & 89.9 & 8.9 & 84.0 & 10.5 & $\uparrow$ & 0.002 \\
\hline ciprofloxacin & 94.6 & 3.1 & 97.2 & 1.7 & 90.8 & 8.0 & - & 0.214 \\
\hline levofloxacin & 92.3 & 4.6 & 90.5 & 2.8 & 83.4 & 11.7 & - & 0.553 \\
\hline erythromycin & 84.6 & 6.9 & 93.3 & 4.5 & 88.6 & 1.5 & - & 0.044 \\
\hline daptomycin & 0 & 100 & 0 & 100 & 0 & 100 & - & 0.139 \\
\hline linezolid & 0.8 & 99.2 & 1.1 & 98.9 & 0.3 & 99.7 & - & 0.931 \\
\hline vancomycin & 1.5 & 98.5 & 0 & 92.2 & 0.6 & 99.1 & - & 0.935 \\
\hline teicoplanin & 0.8 & 99.2 & 0 & 100 & 0.3 & 99.7 & - & 0.807 \\
\hline tigecycline & 0 & 97.7 & 0 & 100 & 0.3 & 98.5 & - & 0.710 \\
\hline \multicolumn{9}{|l|}{ E. faecalis } \\
\hline MDR & 46.7 & - & 55.6 & - & 23.5 & - & - & 0.815 \\
\hline Penicillin G & 12.0 & 88.0 & 8.3 & 91.7 & 6.0 & 94.0 & $\uparrow$ & 0.000 \\
\hline Ampicillin & 18.5 & 81.5 & 2.1 & 97.9 & 5.5 & 94.5 & - & 0.081 \\
\hline Rifampicin & 44.6 & 29.3 & 52.8 & 13.2 & 49.5 & 35.0 & - & NA \\
\hline Ciprofloxacin & 31.5 & 46.7 & 20.8 & 69.4 & 29.0 & 49.0 & - & 0.126 \\
\hline Levofloxacin & 28.3 & 69.6 & 24.3 & 41.0 & 24.0 & 75.5 & $\downarrow$ & 0.000 \\
\hline Erythromycin & 56.5 & 22.8 & 61.1 & 4.9 & 55.5 & 8.0 & $\downarrow$ & 0.010 \\
\hline Daptomycin & 0 & 95.7 & 0 & 98.6 & 0 & 100 & - & 0.137 \\
\hline Linezolid & 0 & 100 & 2.8 & 97.2 & 0.5 & 99.5 & - & 0.710 \\
\hline Vancomycin & 0 & 100 & 0 & 97.2 & 0 & 100 & - & NA \\
\hline Teicoplanin & 1.1 & 98.9 & 0 & 100 & 0 & 100 & $\downarrow$ & 0.019 \\
\hline Tigecycline & 3.3 & 90.2 & 0 & 100 & 0.5 & 99.5 & $\uparrow$ & 0.031 \\
\hline
\end{tabular}




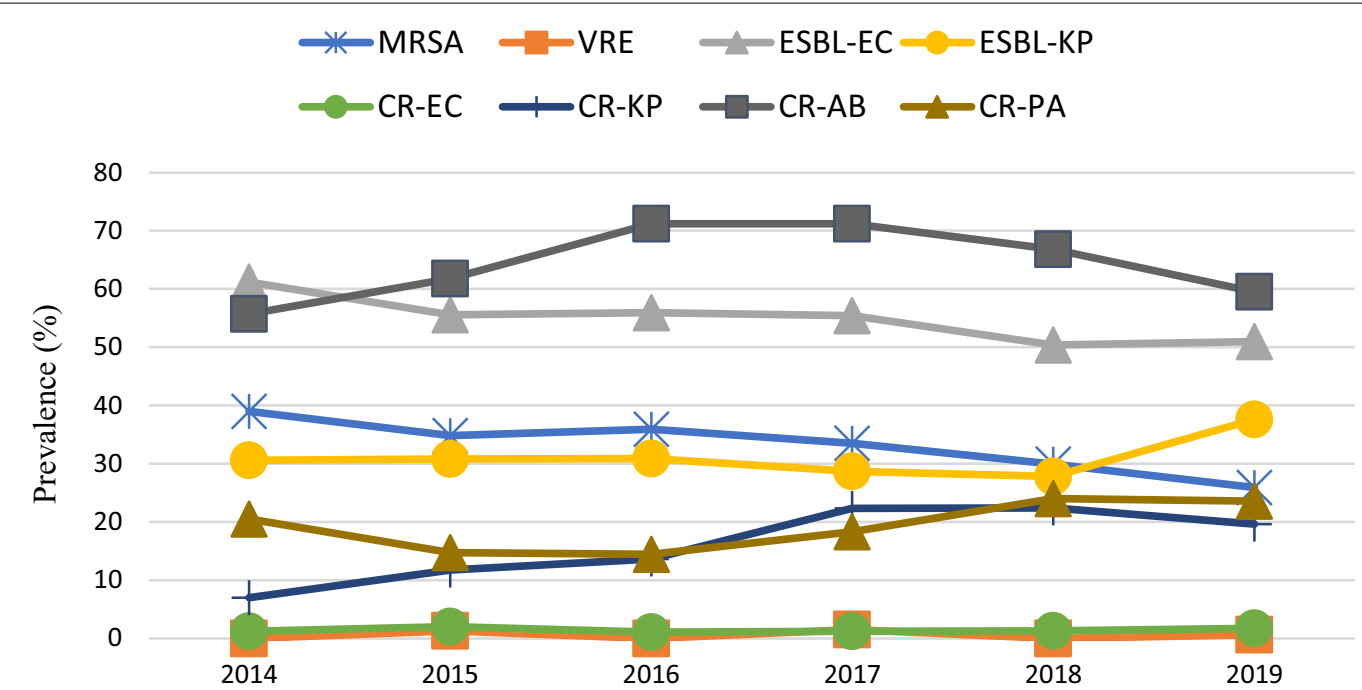

Fig. 2 Trends in the prevalence of major drug-resistant bacteria over the study period. CR-AB, carbapenem-resistant A. baumannii; CR-EC, carbapenem-resistant E. coli; CR-KP, carbapenem-resistant K. pneumoniae; CR-PA, carbapenem-resistant $P$. aeruginosa; ESBL-EC, extended-spectrum $\beta$-lactamase-producing E. coli; ESBL-KP, extended-spectrum $\beta$-lactamase-producing K. pneumoniae; MRSA, methicillin-resistant S. aureus; VRE, vancomycin-resistant E. faecium

and trimethoprim/sulfamethoxazole (Additional file 1: Table S3).

E. faecium showed increased resistance rates to rifampicin over time $(p<0.05)$ (Table 1$)$. Of note, the overall prevalence of vancomycin-resistant E. faecium (VRE) was low. Daptomycin- or linezolid-resistant E. faecium was rare $(\leq 0.1 \%$ and $\leq 0.5 \%$ overall, respectively). The prevalence of MDR E. faecium was similar across different hospital levels and economic regions (Additional file 1: Table S4). 7.4\% E. faecalis were resistant to ampicillin (Table 1).

S. pneumoniae was susceptible to penicillin $\mathrm{G}$ as defined by the breaking point of non-meningitis injection of penicillin G (Additional file 1: Table S5). None of the 388 Streptococcus spp. $\beta$-hemolytic group were resistant to penicillin G, while $1.4 \%$ of $\alpha$-hemolytic Streptococci strains was resistant (Additional file 1: Table S2).

\section{Resistance of Enterobacteriaceae}

Carbapenem-resistant (CR) E. coli (CR-E. coli) and CR $K$. pneumoniae (CR-K. pneumoniae) increased from $1.2 \%$ and $7.0 \%$, respectively, in 2014 to $1.7 \%$ and $19.6 \%$, respectively, in 2019 ( $p=0.397$ and $p<0.05$, respectively) (Table 2, Fig. 2). Unlike E. coli, MDR K. pneumoniae increased significantly over a 6 -year period $(p<0.05)$. Overall, the resistance rate of CR-E. coli to ceftazidime/ avibactam was $60.7 \%$ whereas $8.3 \%$ CR-K. pneumoniae were resistant to ceftazidime/avibactam (Additional file 1: Tables S6 and S7). It is interesting to note that the prevalence of CR-K. pneumonia was higher in tertiary hospitals and developed regions compared with nontertiary hospitals and developing regions (both $p<0.05$ ). However, there was no significant difference in the prevalence of CR-E. coli between developed and developing regions (Additional file 1: Table S4).

The prevalence of ESBL-positive E. coli (ESBL-E. coli) and ESBL-positive $K$. pneumonia (ESBL-K. pneumonia) declined over the period of the study (both $p<0.05$ ) (Table 2, Fig. 2). In general, higher resistance rates of pathogens were more common in tertiary hospitals compared with non-tertiary hospitals (Additional file 1: Table S2). However, the prevalence of ESBL-E. coli and ESBL-K. pneumonia was higher in developing regions compared with developed regions $(58.2 \%$ and $36.4 \%$ vs $48.8 \%$ and $29.6 \%$, respectively; both $p<0.05$ ) (Additional file 1: Table S4).

Other Enterobacteriaceae, including E. cloacae, Klebsiella species (excluding K. pneumoniae), Proteus species, Serratia species, Salmonella species, and Citrobacter species showed lower resistance to ceftazidime/avibactam, carbapenem, and amikacin (Table 2, Additional file 1: Table S5).

\section{Resistance in glucose non-fermenting Gram-negative bacteria}

MDR rates in $A$. baumannii and $P$. aeruginosa were $70.3 \%$ and $21.1 \%$, respectively, which did not increase significantly in 6 years $(p=0.841$ and $p=0.488$, respectively) (Table 3 ). However, the PDR rate in A. baumannii was more than $50 \%(52.5 \%, 558 / 1062)$, wherein $6.7 \%$ 
Table 2 The susceptibility and resistance trends of Gram-negative pathogens to antimicrobial agents

\begin{tabular}{|c|c|c|c|c|c|c|c|c|c|c|c|}
\hline & \multicolumn{5}{|c|}{ All strains (204-2019) } & \multicolumn{2}{|l|}{2014} & \multicolumn{2}{|l|}{2015} & \multicolumn{2}{|c|}{2016} \\
\hline & Number & $\mathrm{MIC}_{50}$ & $\mathrm{MIC}_{90}$ & $\% \mathrm{R}$ & $\% \mathrm{~S}$ & $\% \mathrm{R}$ & $\% \mathrm{~S}$ & $\% \mathrm{R}$ & $\% \mathrm{~S}$ & $\% \mathrm{R}$ & $\% \mathrm{~S}$ \\
\hline \multicolumn{12}{|l|}{ E. coli } \\
\hline MDR & 9944 & - & - & 71.4 & - & 76.2 & - & 65.8 & - & 72.1 & - \\
\hline ESBL-E. coli & 9944 & - & - & 53.4 & - & 61.2 & - & 55.6 & - & 55.9 & - \\
\hline CR-E. coli & 9944 & - & - & 1.5 & - & 1.2 & - & 2.0 & - & 1.1 & - \\
\hline amoxicillin & 9944 & 128 & 256 & 74.8 & 20.4 & 93.2 & 5.1 & 83.4 & 15.8 & 84.9 & 14.4 \\
\hline amoxicillin/clavulanic acid & 9944 & 16 & 256 & 39.3 & 40.6 & 26.9 & 40.6 & 14.6 & 60.7 & 18.0 & 56.4 \\
\hline piperacillin/tazobactam & 9944 & 2 & 64 & 7.5 & 86.4 & 12.3 & 78.0 & 8.1 & 88.3 & 6.7 & 89.1 \\
\hline cefoperazone/sulbactamª & 9944 & 4 & 32 & 8.5 & 80.6 & 15.3 & 65.5 & 9.3 & 76.5 & 8.0 & 79.5 \\
\hline ceftazidime/avibactam & 3683 & 0.25 & 1 & 1.0 & 99.0 & - & - & - & - & - & - \\
\hline cefazolin & 9944 & 128 & 128 & 59.7 & 34.8 & 69.1 & 26.7 & 62.6 & 30.7 & 63.5 & 29.8 \\
\hline cefuroxime & 9944 & 128 & 128 & 55.0 & 41.5 & 64.8 & 31.8 & 58.7 & 40.1 & 59.9 & 37.4 \\
\hline ceftazidime & 9944 & 1 & 64 & 28.9 & 63.2 & 33.9 & 58.7 & 30.6 & 62.3 & 30.9 & 61.9 \\
\hline ceftriaxone & 9944 & 16 & 64 & 55.8 & 43.8 & 65.8 & 33.7 & 57.8 & 41.9 & 59.6 & 40.3 \\
\hline cefepime & 9944 & 1 & 32 & 21.0 & 58.4 & 30.2 & 46.7 & 24.4 & 54.1 & 25.8 & 51.7 \\
\hline cefoxitin & 9944 & 4 & 32 & 13.6 & 77.6 & 19.5 & 69.4 & 13.5 & 80.4 & 13.9 & 74.6 \\
\hline moxalactam & 9943 & 0.25 & 2 & 2.5 & 95.6 & 3.0 & 93.9 & 2.3 & 94.4 & 2.6 & 94.9 \\
\hline aztreonam & 9944 & 4 & 64 & 36.2 & 55.6 & 42.2 & 49.5 & 37.5 & 54.8 & 37.6 & 53.6 \\
\hline ertapenem & 5495 & 0.016 & 0.064 & 1.5 & 97.9 & - & - & - & - & - & - \\
\hline imipenem & 9944 & 0.125 & 0.5 & 1.4 & 98.3 & 1.0 & 97.8 & 1.4 & 98.1 & 1.1 & 98.7 \\
\hline meropenem & 9944 & 0.016 & 0.064 & 1.4 & 98.5 & 1.2 & 98.7 & 1.5 & 98.2 & 1.1 & 98.7 \\
\hline amikacin & 9944 & 4 & 8 & 2.9 & 96.7 & 4.9 & 93.8 & 3.3 & 96.3 & 3.6 & 95.5 \\
\hline gentamicin & 9944 & 4 & 128 & 39.5 & 58.7 & 44.0 & 51.9 & 39.9 & 57.1 & 43.3 & 53.6 \\
\hline ciprofloxacin & 9944 & 4 & 32 & 64.3 & 26.0 & 65.7 & 27.0 & 58.9 & 30.9 & 74.0 & 17.9 \\
\hline levofloxacin & 9944 & 4 & 32 & 53.3 & 37.1 & 61.7 & 31.6 & 53.1 & 37.2 & 57.5 & 34.3 \\
\hline trimethoprim/sulfamethoxazole & 9944 & 8 & 8 & 57.1 & 42.9 & 60.8 & 39.2 & 58.3 & 41.7 & 58.7 & 41.3 \\
\hline fosfomycin & 9944 & 0.5 & 4 & 1.7 & 95.2 & 0.1 & 93.2 & 0.2 & 95.5 & 0.6 & 94.5 \\
\hline polymyxin B & 9944 & 0.5 & 2 & 2.2 & 97.8 & 1.5 & 98.5 & 1.4 & 98.6 & 7.2 & 92.8 \\
\hline tigecycline & 9944 & 0.25 & 0.5 & 0 & 100 & 0 & 99.6 & 0 & 100 & 0 & 100 \\
\hline \multicolumn{12}{|l|}{ K.pneumoniae } \\
\hline MDR & 4378 & - & - & 38.7 & - & 30.6 & - & 32.6 & - & 36.1 & - \\
\hline ESBL-K. pneumoniae & 4378 & - & - & 32.5 & - & 30.6 & - & 30.8 & - & 30.9 & - \\
\hline CR-K.pneumoniae & 4378 & - & - & 18.3 & - & 7.0 & - & 11.7 & - & 13.6 & - \\
\hline amoxicillin/clavulanic acid & 3679 & 8 & 128 & 36.0 & 52.4 & 29.7 & 45.4 & 21.4 & 67.7 & 32.5 & 56.7 \\
\hline piperacillin/tazobactam & 4378 & 4 & 128 & 22.0 & 70.5 & 14.0 & 81.2 & 15.5 & 79.5 & 20.1 & 75.5 \\
\hline cefoperazone/sulbactamª & 4378 & 1 & 128 & 24.9 & 69.7 & 14.4 & 76.9 & 19.4 & 73.9 & 22.3 & 71.5 \\
\hline ceftazidime/avibactam & 1655 & 0.5 & 4 & 1.6 & 98.4 & - & - & - & - & - & - \\
\hline cefazolin & 4378 & 2 & 128 & 43.5 & 52.5 & 40.6 & 55.9 & 40.2 & 55.1 & 43.3 & 50.4 \\
\hline cefuroxime & 4378 & 8 & 128 & 42.7 & 54.7 & 37.1 & 61.1 & 37.5 & 59.5 & 43.1 & 54.7 \\
\hline ceftazidime & 4378 & 0.5 & 64 & 31.6 & 64.9 & 20.1 & 73.8 & 27.0 & 69.8 & 30.9 & 64.8 \\
\hline ceftriaxone & 4378 & 0.125 & 64 & 40.2 & 59.3 & 34.5 & 65.5 & 37.5 & 62.2 & 39.5 & 60.0 \\
\hline cefepime & 4378 & 0.125 & 64 & 27 & 64.8 & 16.2 & 72.9 & 20.8 & 70.7 & 26.5 & 65.5 \\
\hline cefoxitin & 4378 & 4 & 128 & 27.3 & 68.6 & 22.3 & 75.5 & 20.5 & 73.6 & 25.5 & 70.1 \\
\hline moxalactam & 4378 & 0.5 & 128 & 17.4 & 80.3 & 6.6 & 92.1 & 10.0 & 87.1 & 14.3 & 83 \\
\hline aztreonam & 4378 & 0.125 & 64 & 34.1 & 63.7 & 24.0 & 72.9 & 29.0 & 70.4 & 32.5 & 65.5 \\
\hline ertapenem & 2518 & 0.016 & 32 & 20.5 & 78.6 & - & - & - & - & - & - \\
\hline imipenem & 4378 & 0.25 & 32 & 17.5 & 81.6 & 5.2 & 93.9 & 9.7 & 88.9 & 13.2 & 86.0 \\
\hline meropenem & 4378 & 0.032 & 32 & 17.4 & 82.2 & 5.7 & 93.4 & 9.7 & 89.1 & 12.6 & 86.8 \\
\hline amikacin & 4378 & 2 & 128 & 13.7 & 86.2 & 6.1 & 93.9 & 9.1 & 90.9 & 12.2 & 87.5 \\
\hline gentamicin & 4378 & 1 & 128 & 26.8 & 72.4 & 20.1 & 78.6 & 21.1 & 78.0 & 26.5 & 72.6 \\
\hline ciprofloxacin & 4378 & 0.25 & 32 & 44.5 & 51.7 & 36.7 & 58.5 & 40.2 & 56.3 & 43.3 & 50.4 \\
\hline levofloxacin & 4378 & 0.25 & 32 & 34.1 & 55.8 & 25.3 & 60.7 & 26.1 & 61.9 & 31.4 & 58.0 \\
\hline
\end{tabular}


Table 2 (continued)

\begin{tabular}{|c|c|c|c|c|c|c|c|c|c|c|c|}
\hline & \multicolumn{5}{|c|}{ All strains (204-2019) } & \multicolumn{2}{|l|}{2014} & \multicolumn{2}{|c|}{2015} & \multicolumn{2}{|l|}{2016} \\
\hline & Number & $\mathrm{MIC}_{50}$ & $\mathrm{MIC}_{90}$ & $\% \mathrm{R}$ & $\% \mathrm{~S}$ & $\% \mathrm{R}$ & $\% \mathrm{~S}$ & $\% \mathrm{R}$ & $\% \mathrm{~S}$ & $\% \mathrm{R}$ & $\% \mathrm{~S}$ \\
\hline trimethoprim/sulfamethoxazole & 4378 & 0.125 & 8 & 36.8 & 63.2 & 31.9 & 68.1 & 35.8 & 64.2 & 32.5 & 67.5 \\
\hline fosfomycin & 4378 & 4 & 128 & 6.1 & 89.6 & 0.4 & 90.8 & 0.6 & 90.0 & 2.9 & 91.7 \\
\hline polymyxin B & 4378 & 1 & 2 & 3.6 & 96.4 & 0.9 & 99.1 & 1.5 & 98.5 & 5.7 & 94.3 \\
\hline tigecycline & 4378 & 0.5 & 1 & 0.2 & 98.9 & 0.9 & 95.2 & 1.5 & 97.4 & 0.1 & 99.1 \\
\hline \multicolumn{12}{|l|}{ E. cloacae } \\
\hline MDR & - & - & - & 20.8 & - & 24.6 & & 28.9 & & 23.8 & \\
\hline piperacillin/tazobactam & 785 & 2 & 128 & 13.1 & 78.5 & 9.2 & 84.6 & 11.8 & 80.3 & 14.6 & 76.9 \\
\hline cefoperazone/sulbactama & 785 & 0.5 & 32 & 9.8 & 80.4 & 7.7 & 83.1 & 15.8 & 75.0 & 8.5 & 76.9 \\
\hline ceftazidime/avibactam & 265 & 0.25 & 4 & 6.0 & 94.0 & - & - & - & - & - & - \\
\hline ceftazidime & 785 & 0.25 & 64 & 29.6 & 67.5 & 27.7 & 69.2 & 23.7 & 72.4 & 28.5 & 69.2 \\
\hline ceftriaxone & 785 & 0.25 & 64 & 39.9 & 59.5 & 52.3 & 46.2 & 42.1 & 57.9 & 40.8 & 59.2 \\
\hline cefepime & 785 & 0.125 & 16 & 13.4 & 78.7 & 6.2 & 84.6 & 13.2 & 81.6 & 12.3 & 75.4 \\
\hline moxalactam & 785 & 0.25 & 32 & 6.2 & 84.2 & 3.1 & 86.2 & 5.3 & 81.6 & 6.9 & 83.1 \\
\hline aztreonam & 785 & 0.125 & 64 & 29.7 & 68.4 & 27.7 & 67.7 & 25.0 & 75.0 & 29.2 & 69.2 \\
\hline ertapenem & 423 & 0.016 & 0.5 & 5.4 & 92.4 & - & - & - & - & - & - \\
\hline imipenem & 785 & 0.25 & 1 & 4.8 & 93.0 & 1.5 & 90.8 & 5.3 & 92.1 & 4.6 & 92.3 \\
\hline meropenem & 785 & 0.032 & 0.125 & 4.5 & 94.6 & 0 & 98.5 & 3.9 & 94.7 & 4.6 & 91.5 \\
\hline amikacin & 785 & 2 & 4 & 2.3 & 97.3 & 4.6 & 95.4 & 1.3 & 98.7 & 3.8 & 95.4 \\
\hline gentamicin & 785 & 1 & 64 & 14.5 & 81.7 & 12.3 & 83.1 & 11.8 & 77.6 & 13.8 & 83.8 \\
\hline ciprofloxacin & 785 & 0.064 & 16 & 25.0 & 69.3 & 24.6 & 63.1 & 26.3 & 69.7 & 25.4 & 67.7 \\
\hline levofloxacin & 785 & 0.064 & 8 & 17.7 & 73.8 & 23.1 & 69.2 & 21.1 & 69.7 & 17.7 & 73.8 \\
\hline trimethoprim/sulfamethoxazole & 785 & 0.125 & 8 & 25.1 & 74.9 & 41.5 & 58.5 & 31.6 & 68.4 & 19.2 & 80.8 \\
\hline fosfomycin & 785 & 8 & 64 & 1.1 & 93.2 & 0 & 84.6 & 0 & 90.8 & 0.8 & 93.8 \\
\hline polymyxin B & 785 & 1 & 32 & 28.7 & 71.3 & 1.5 & 98.5 & 0 & 100 & 20.0 & 80.0 \\
\hline tigecycline & 785 & 0.25 & 1 & 0.8 & 98.2 & 1.5 & 98.5 & 0.5 & 98.2 & 2.3 & 96.9 \\
\hline \multicolumn{12}{|l|}{ Klebsiella spp } \\
\hline MDR & 401 & - & - & 20.0 & - & 44.4 & & 21.9 & & 35.4 & \\
\hline Piperacillin/tazobactam & 401 & 1 & 128 & 15.5 & 79.3 & 33.3 & 63.0 & 9.4 & 90.6 & 19.0 & 78.5 \\
\hline Cefoperazone/Sulbactama & 401 & 4 & 128 & 18.0 & 74.1 & 14.8 & 77.8 & 9.4 & 84.4 & 12.7 & 74.7 \\
\hline Ceftazidime/avibactam & 130 & 0.25 & 4 & 4.6 & 95.4 & - & - & - & - & - & - \\
\hline Cefazolin & 198 & 4 & 128 & 43.9 & 37.9 & 72.7 & 18.2 & 40.9 & 45.5 & 60.5 & 32.6 \\
\hline Cefuroxime & 266 & 8 & 128 & 38.0 & 58.3 & 59.3 & 37.0 & 31.2 & 62.5 & 62.8 & 34.9 \\
\hline Ceftazidime & 401 & 0.5 & 64 & 28.7 & 68.3 & 37.0 & 48.1 & 18.8 & 81.2 & 32.9 & 64.6 \\
\hline Ceftriaxone & 401 & 0.25 & 64 & 39.4 & 58.9 & 63.0 & 37.0 & 25.0 & 75.0 & 50.6 & 48.1 \\
\hline Cefepime & 401 & 0.125 & 16 & 15.0 & 79.1 & 18.5 & 63.0 & 12.5 & 81.2 & 22.8 & 68.4 \\
\hline Cefoxitin & 198 & 4 & 128 & 18.2 & 76.8 & 54.5 & 45.5 & 18.2 & 81.8 & 16.3 & 76.7 \\
\hline Moxalactam & 400 & 0.25 & 16 & 7.5 & 88.5 & 7.4 & 88.9 & 3.1 & 93.8 & 6.3 & 89.9 \\
\hline Aztreonam & 401 & 0.25 & 64 & 28.7 & 67.8 & 51.9 & 37.0 & 21.9 & 78.1 & 31.6 & 62.0 \\
\hline Ertapenem & 204 & 0.016 & 1 & 7.4 & 89.2 & - & - & - & - & - & - \\
\hline Imipenem & 401 & 0.25 & 2 & 6.2 & 89.8 & 3.7 & 88.9 & 3.1 & 96.9 & 5.1 & 89.9 \\
\hline Meropenem & 401 & 0.032 & 0.25 & 5.0 & 94.0 & 0 & 100 & 3.1 & 96.9 & 3.8 & 93.7 \\
\hline Amikacin & 401 & 2 & 8 & 2.5 & 97.3 & 7.4 & 92.6 & 3.1 & 96.9 & 3.8 & 94.9 \\
\hline Gentamicin & 401 & 1 & 64 & 12.0 & 86.0 & 14.8 & 85.2 & 12.5 & 78.1 & 26.6 & 70.9 \\
\hline Ciprofloxacin & 401 & 0.064 & 32 & 30.2 & 67.3 & 48.1 & 48.1 & 31.2 & 68.8 & 49.4 & 49.4 \\
\hline Levofloxacin & 401 & 0.125 & 8 & 21.2 & 72.1 & 44.4 & 51.9 & 21.9 & 68.8 & 30.4 & 58.2 \\
\hline Trimethoprim/Sulfamethoxazole & 401 & 0.125 & 8 & 19.0 & 81.0 & 37.0 & 63.0 & 31.2 & 68.8 & 27.8 & 72.2 \\
\hline Fosfomycin & 401 & 4 & 64 & 2.5 & 93.5 & 0 & 92.6 & 0 & 84.4 & 2.5 & 93.7 \\
\hline Polymyxin B & 401 & 1 & 2 & 6.0 & 94.0 & 0 & 100 & 0 & 100 & 5.1 & 94.9 \\
\hline Tigecycline & 401 & 0.25 & 1 & 1.2 & 97.8 & 7.4 & 92.6 & 6.2 & 93.8 & 1.3 & 96.2 \\
\hline \multicolumn{12}{|l|}{ Salmonella spp } \\
\hline MDR & - & - & - & 45.1 & - & 36.4 & - & 29.4 & - & 38.7 & - \\
\hline
\end{tabular}


Table 2 (continued)

\begin{tabular}{|c|c|c|c|c|c|c|c|c|c|c|c|}
\hline & \multicolumn{5}{|c|}{ All strains (204-2019) } & \multicolumn{2}{|l|}{2014} & \multicolumn{2}{|l|}{2015} & \multicolumn{2}{|l|}{2016} \\
\hline & Number & $\mathrm{MIC}_{50}$ & $\mathrm{MIC}_{90}$ & $\% \mathrm{R}$ & $\% \mathrm{~S}$ & $\% R$ & $\% \mathrm{~S}$ & $\% R$ & $\% \mathrm{~S}$ & $\% \mathrm{R}$ & $\% \mathrm{~S}$ \\
\hline Amoxicillin & 384 & 128 & 256 & 57.6 & 40.6 & 57.6 & 39.4 & 50.0 & 50.0 & 37.1 & 59.7 \\
\hline Amoxicillin/Clavulanic Acid & 384 & 8 & 128 & 7.6 & 91.6 & 8.2 & 57.6 & 5.9 & 82.4 & 4.5 & 35.5 \\
\hline Piperacillin/Tazobactam & 384 & 1 & 16 & 1.6 & 95.8 & 0 & 87.9 & 5.9 & 100 & 1.6 & 100 \\
\hline Cefoperazone/Sulbactam ${ }^{\mathrm{a}}$ & 384 & 2 & 8 & 2.1 & 95.8 & 9.1 & 90.9 & 0 & 94.1 & 0 & 98.4 \\
\hline Ceftazidime/Avibactam & 117 & 0.5 & 0.5 & 0 & 100 & - & - & - & - & - & - \\
\hline Ceftazidime & 384 & 0.25 & 4 & 9.9 & 90.1 & 12.1 & 87.9 & 8.8 & 91.2 & 4.8 & 95.2 \\
\hline Ceftriaxone & 384 & 0.125 & 16 & 10.9 & 88.8 & 15.2 & 84.8 & 5.9 & 91.2 & 6.5 & 93.5 \\
\hline Cefepime & 384 & 0.125 & 1 & 5.7 & 91.4 & 0 & 90.9 & 2.9 & 97.1 & 6.5 & 93.5 \\
\hline Moxalactam & 384 & 0.25 & 8 & 1.0 & 95.6 & 0 & 100 & 0 & 94.1 & 1.6 & 93.5 \\
\hline Aztreonam & 384 & 0.125 & 8 & 6.2 & 84.9 & 12.1 & 84.8 & 2.9 & 97.1 & 1.6 & 74.2 \\
\hline Ertapenem & 201 & 0.008 & 0.016 & 0.5 & 99.5 & - & - & - & - & - & - \\
\hline Imipenem & 384 & 0.064 & 0.25 & 0.3 & 99.2 & 0 & 100 & 0 & 97.1 & 0 & 100 \\
\hline Meropenem & 384 & 0.032 & 0.125 & 1.6 & 96.4 & 3.0 & 97.0 & 0 & 100 & 1.1 & 91.9 \\
\hline Amikacin & 384 & 2 & 8 & 1.0 & 98.7 & 3.0 & 97.0 & 0 & 100 & 0 & 100 \\
\hline Gentamicin & 384 & 1 & 4 & 7.3 & 91.9 & 9.1 & 90.9 & 2.9 & 97.1 & 6.5 & 93.5 \\
\hline Ciprofloxacin & 384 & 0.5 & 1 & 18 & 29.7 & 18.2 & 36.4 & 14.7 & 35.3 & 25.8 & 17.7 \\
\hline Levofloxacin & 384 & 0.25 & 1 & 8.6 & 33.1 & 6.1 & 33.3 & 5.9 & 32.4 & 3.2 & 30.6 \\
\hline Trimethoprim/Sulfamethoxazole & 384 & 0.125 & 8 & 13.8 & 86.2 & 15.2 & 84.8 & 11.8 & 88.2 & 4.8 & 95.2 \\
\hline Fosfomycin & 384 & 0.5 & 4 & 0.5 & 99.0 & 0 & 97.0 & 0 & 100 & 0 & 100 \\
\hline Polymyxin B & 384 & 2 & 16 & 38.3 & 61.7 & 0 & 100 & 0 & 100 & 37.1 & 62.9 \\
\hline Tigecycline & 384 & 0.25 & 1 & 1.3 & 97.4 & 0 & 100 & 2.9 & 97.1 & 0 & 91.9 \\
\hline \multicolumn{12}{|l|}{ Serratia spp } \\
\hline MDR & - & - & - & 9.3 & - & 37.5 & - & 10.3 & - & 11.9 & - \\
\hline Piperacillin/Tazobactam & 313 & 1 & 64 & 6.4 & 87.2 & 25.0 & 75.0 & 6.9 & 82.8 & 6.0 & 85.1 \\
\hline Cefoperazone/Sulbactama & 312 & 1 & 64 & 16.0 & 75.6 & 25.0 & 50.0 & 17.2 & 72.4 & 4.5 & 82.1 \\
\hline Ceftazidime/Avibactam & 83 & 0.125 & 0.25 & 0 & 100 & - & - & - & - & - & - \\
\hline Ceftazidime & 311 & 0.25 & 8 & 9.6 & 89.1 & 37.5 & 62.5 & 6.9 & 89.7 & 10.4 & 88.1 \\
\hline Ceftriaxone & 312 & 0.125 & 64 & 28.8 & 71.2 & 37.5 & 62.5 & 31.0 & 69.0 & 25.4 & 74.6 \\
\hline Cefepime & 313 & 0.125 & 16 & 18.5 & 74.8 & 25.0 & 75.0 & 20.7 & 72.4 & 11.9 & 80.6 \\
\hline Moxalactam & 312 & 0.5 & 4 & 3.2 & 94.9 & 12.5 & 87.5 & 0 & 100 & 1.5 & 94.0 \\
\hline Aztreonam & 313 & 0.125 & 32 & 16.0 & 72.8 & 25.0 & 75.0 & 24.1 & 75.9 & 19.4 & 76.1 \\
\hline Ertapenem & 169 & 0.016 & 0.064 & 5.3 & 94.1 & - & - & - & - & - & - \\
\hline Imipenem & 313 & 0.5 & 2 & 7.0 & 89.1 & 37.5 & 62.5 & 3.4 & 96.6 & 3.0 & 94.0 \\
\hline Meropenem & 313 & 0.064 & 0.25 & 5.1 & 93.0 & 25.0 & 75.0 & 0 & 96.6 & 1.5 & 94.0 \\
\hline Amikacin & 313 & 4 & 16 & 1.0 & 98.4 & 0 & 100 & 0 & 100 & 0 & 97.0 \\
\hline Gentamicin & 313 & 2 & 128 & 20.1 & 79.2 & 25.0 & 62.5 & 20.7 & 79.3 & 19.4 & 79.1 \\
\hline Ciprofloxacin & 312 & 0.25 & 16 & 30.4 & 50.0 & 25.0 & 50.0 & 31.0 & 69.0 & 29.9 & 47.8 \\
\hline Levofloxacin & 313 & 0.5 & 16 & 25.9 & 68.1 & 25.0 & 62.5 & 20.7 & 75.9 & 25.4 & 70.1 \\
\hline Trimethoprim/Sulfamethoxazole & 313 & 0.125 & 0.5 & 6.1 & 93.9 & 37.5 & 62.5 & 10.3 & 89.7 & 11.9 & 88.1 \\
\hline Fosfomycin & 312 & 4 & 8 & 0.6 & 98.7 & 0 & 100 & 0 & 100 & 0 & 100 \\
\hline \multirow[t]{3}{*}{ Tigecycline } & 312 & 0.5 & 2 & 1.5 & 97.5 & 7.5 & 92.5 & 1.7 & 98.3 & 1.5 & 98.5 \\
\hline & \multicolumn{2}{|c|}{2017} & \multicolumn{3}{|c|}{2018} & \multicolumn{3}{|c|}{2019} & \multirow{2}{*}{\multicolumn{2}{|c|}{ Trend }} & \multirow[t]{2}{*}{$p$} \\
\hline & $\% R$ & $\% \mathrm{~S}$ & & & $\% \mathrm{~S}$ & $\% \mathrm{R}$ & & $\% \mathrm{~S}$ & & & \\
\hline \multicolumn{12}{|l|}{ E. coli } \\
\hline MDR & 71.2 & - & \multicolumn{2}{|c|}{71.1} & - & \multicolumn{2}{|c|}{71.8} & - & \multicolumn{2}{|l|}{-} & 0.749 \\
\hline ESBL-E. coli & 55.4 & - & \multicolumn{2}{|c|}{50.4} & - & 51. & & - & $\downarrow$ & & 0.000 \\
\hline CR-E. coli & 1.2 & - & & & - & 1.7 & & - & - & & 0.397 \\
\hline amoxicillin & 85.6 & 13.9 & & & 14.5 & 83. & & 15.0 & $\downarrow$ & & 0.000 \\
\hline amoxicillin/clavulanic acid & 19.7 & 51.5 & & & 48.7 & 44. & & 38.1 & $\uparrow$ & & 0.000 \\
\hline piperacillin/tazobactam & 6.2 & 89.0 & & & 82.5 & 8.1 & & 83.0 & - & & 0.04 \\
\hline
\end{tabular}


Table 2 (continued)

\begin{tabular}{|c|c|c|c|c|c|c|c|c|}
\hline & \multicolumn{2}{|c|}{2017} & \multicolumn{2}{|l|}{2018} & \multicolumn{2}{|c|}{2019} & \multirow[t]{2}{*}{ Trend } & \multirow[t]{2}{*}{$p$} \\
\hline & $\% R$ & $\% \mathrm{~S}$ & $\% R$ & $\% \mathrm{~S}$ & $\% R$ & $\% \mathrm{~S}$ & & \\
\hline cefoperazone/sulbactam ${ }^{a}$ & 8.7 & 80.7 & 7.0 & 91.1 & 7.8 & 84.0 & $\downarrow$ & 0.000 \\
\hline ceftazidime/avibactam & - & - & - & - & 1.0 & 99.0 & - & NA \\
\hline cefazolin & 61.8 & 31.2 & 57.1 & 37.0 & 56.1 & 39.6 & $\uparrow$ & 0.000 \\
\hline cefuroxime & 50.6 & 45.4 & 53.6 & 43.0 & 52.7 & 43.1 & $\downarrow$ & 0.000 \\
\hline ceftazidime & 28.9 & 62.6 & 25.6 & 65.8 & 28.2 & 63.7 & $\downarrow$ & 0.000 \\
\hline ceftriaxone & 57.3 & 42.1 & 53.5 & 46.0 & 52.6 & 47.2 & $\downarrow$ & 0.000 \\
\hline cefepime & 24.9 & 52.4 & 20.6 & 58.5 & 15.3 & 66.6 & $\downarrow$ & 0.000 \\
\hline cefoxitin & 17.0 & 74.4 & 9.5 & 82.9 & 13.1 & 78.1 & $\downarrow$ & 0.000 \\
\hline moxalactam & 2.5 & 95.5 & 2.3 & 96.8 & 2.5 & 95.9 & - & 0.661 \\
\hline aztreonam & 34.9 & 54.8 & 30.9 & 59.8 & 37.3 & 55.9 & $\downarrow$ & 0.021 \\
\hline ertapenem & - & - & 1.3 & 98.2 & 1.7 & 97.7 & - & 0.281 \\
\hline imipenem & 1.2 & 98.4 & 1.3 & 98.5 & 1.6 & 98.2 & - & 0.140 \\
\hline meropenem & 1.0 & 98.6 & 1.3 & 98.6 & 1.6 & 98.4 & - & 0.217 \\
\hline amikacin & 3.1 & 96.3 & 2.5 & 97.2 & 2.3 & 97.7 & $\downarrow$ & 0.000 \\
\hline gentamicin & 43.5 & 54.0 & 40.9 & 58.1 & 34.9 & 64.4 & $\downarrow$ & 0.000 \\
\hline ciprofloxacin & 64.5 & 24.4 & 66.3 & 25.1 & 60.5 & 28.9 & $\downarrow$ & 0.000 \\
\hline levofloxacin & 54.4 & 36.0 & 51.1 & 38.4 & 50.9 & 38.9 & $\downarrow$ & 0.000 \\
\hline trimethoprim/sulfamethoxazole & 60.3 & 39.7 & 57.7 & 42.3 & 54.0 & 46.0 & $\downarrow$ & 0.000 \\
\hline fosfomycin & 0.4 & 95.1 & 2.2 & 95.4 & 3.1 & 95.7 & $\uparrow$ & 0.000 \\
\hline polymyxin B & 0.5 & 99.5 & 3.1 & 96.9 & 0.7 & 99.3 & $\downarrow$ & 0.000 \\
\hline tigecycline & 0 & 100 & 0 & 100 & 0 & 100 & - & NA \\
\hline \multicolumn{9}{|l|}{ K.pneumoniae } \\
\hline MDR & 42.2 & - & 40.7 & - & 39.9 & - & $\uparrow$ & 0.000 \\
\hline ESBL-K. pneumoniae & 28.7 & - & 27.8 & - & 27.6 & - & $\downarrow$ & 0.001 \\
\hline CR-K.pneumoniae & 22.3 & - & 22.4 & - & 19.6 & - & $\uparrow$ & 0.000 \\
\hline amoxicillin/clavulanic acid & 31.8 & 58.8 & 37.0 & 55.0 & 40.9 & 46.5 & $\uparrow$ & 0.000 \\
\hline piperacillin/tazobactam & 25.8 & 70.6 & 17.5 & 70.2 & 26.2 & 65.2 & $\uparrow$ & 0.000 \\
\hline cefoperazone/sulbactamª & 27.5 & 67.6 & 27.3 & 66.4 & 26.3 & 69.5 & $\uparrow$ & 0.000 \\
\hline ceftazidime/avibactam & - & - & - & - & 1.6 & 98.4 & - & NA \\
\hline cefazolin & 48.5 & 47.6 & 44.7 & 52.1 & 42.4 & 54.3 & - & 0.723 \\
\hline cefuroxime & 45.3 & 51.9 & 43.7 & 53.7 & 42.9 & 54.3 & - & 0.094 \\
\hline ceftazidime & 34.8 & 62 & 32.9 & 62.9 & 32.5 & 64.9 & $\uparrow$ & 0.001 \\
\hline ceftriaxone & 44.6 & 54.9 & 41.3 & 57.7 & 39.6 & 59.8 & - & 0.309 \\
\hline cefepime & 33.4 & 58.4 & 32.0 & 61.9 & 25.1 & 66.0 & - & 0.03 \\
\hline cefoxitin & 29.9 & 66.4 & 30.1 & 66.9 & 27.7 & 67.7 & $\uparrow$ & 0.004 \\
\hline moxalactam & 21.1 & 76.4 & 20.4 & 76.4 & 18.9 & 79.5 & $\uparrow$ & 0.000 \\
\hline aztreonam & 36.7 & 60.0 & 36.0 & 61.8 & 35.3 & 62.7 & $\uparrow$ & 0.000 \\
\hline ertapenem & - & - & 22.2 & 77.1 & 19.6 & 79.5 & - & 0.115 \\
\hline imipenem & 21.1 & 77.4 & 21.4 & 77.6 & 19.4 & 80.1 & $\uparrow$ & 0.000 \\
\hline meropenem & 20.3 & 78.9 & 21.4 & 78.3 & 19.5 & 80.4 & $\uparrow$ & 0.000 \\
\hline amikacin & 16.7 & 83.1 & 14.6 & 85.2 & 14.7 & 85.1 & $\uparrow$ & 0.000 \\
\hline gentamicin & 31.6 & 67.1 & 27.7 & 71.7 & 26.8 & 72.6 & - & 0.036 \\
\hline ciprofloxacin & 51.7 & 46.6 & 44.0 & 50.3 & 44.7 & 52.8 & - & 0.065 \\
\hline levofloxacin & 39.5 & 51.0 & 34.9 & 53.9 & 35.7 & 55.5 & $\uparrow$ & 0.000 \\
\hline trimethoprim/sulfamethoxazole & 44.6 & 55.4 & 36.3 & 63.7 & 37.0 & 63.0 & - & 0.147 \\
\hline fosfomycin & 5.7 & 89.2 & 9.4 & 86.2 & 7.7 & 90.3 & $\uparrow$ & 0.000 \\
\hline polymyxin B & 1.0 & 99.0 & 9.0 & 91.0 & 1.6 & 98.4 & - & 0.937 \\
\hline tigecycline & 0 & 98.8 & 0.1 & 98.6 & 0.1 & 99.9 & $\downarrow$ & 0.001 \\
\hline
\end{tabular}


Table 2 (continued)

\begin{tabular}{|c|c|c|c|c|c|c|c|c|}
\hline & \multicolumn{2}{|c|}{2017} & \multicolumn{2}{|c|}{2018} & \multicolumn{2}{|c|}{2019} & \multirow[t]{2}{*}{ Trend } & \multirow[t]{2}{*}{$p$} \\
\hline & $\% R$ & $\% \mathrm{~S}$ & $\% R$ & $\% \mathrm{~S}$ & $\% R$ & $\% \mathrm{~S}$ & & \\
\hline \multicolumn{9}{|l|}{ E. cloacae } \\
\hline MDR & 16.5 & & 12.0 & & 22.6 & & - & 0.142 \\
\hline piperacillin/tazobactam & 4.4 & 75.8 & 16.5 & 75.9 & 14.7 & 79.6 & - & 0.176 \\
\hline cefoperazone/sulbactamª & 4.4 & 79.1 & 10.8 & 82.9 & 10.6 & 81.9 & - & 0.868 \\
\hline ceftazidime/avibactam & - & - & - & - & 6.0 & 94.0 & - & NA \\
\hline ceftazidime & 33.0 & 62.6 & 27.2 & 70.3 & 32.5 & 64.9 & - & 0.229 \\
\hline ceftriaxone & 44.0 & 56.0 & 32.9 & 66.5 & 38.5 & 60.4 & - & 0.039 \\
\hline cefepime & 11.0 & 82.4 & 13.3 & 82.3 & 16.6 & 74.7 & - & 0.039 \\
\hline moxalactam & 6.6 & 83.5 & 4.4 & 86.7 & 7.9 & 83.8 & - & 0.256 \\
\hline aztreonam & 36.3 & 61.5 & 27.8 & 71.5 & 30.6 & 66.8 & - & 0.531 \\
\hline ertapenem & - & - & 4.4 & 93.7 & 6.0 & 91.7 & - & 0.481 \\
\hline imipenem & 6.6 & 93.4 & 4.4 & 92.4 & 5.3 & 94.3 & - & 0.437 \\
\hline meropenem & 5.5 & 94.5 & 3.8 & 96.2 & 5.7 & 94.3 & - & 0.130 \\
\hline amikacin & 2.2 & 96.7 & 0.6 & 99.4 & 2.3 & 97.4 & - & 0.262 \\
\hline gentamicin & 14.3 & 79.1 & 11.4 & 87.3 & 18.1 & 78.9 & - & 0.156 \\
\hline ciprofloxacin & 24.2 & 68.1 & 21.5 & 72.2 & 26.8 & 70.2 & - & 0.909 \\
\hline levofloxacin & 17.6 & 75.8 & 17.7 & 77.2 & 15.5 & 73.2 & - & 0.125 \\
\hline trimethoprim/sulfamethoxazole & 26.4 & 73.6 & 15.2 & 84.8 & 27.5 & 72.5 & - & 0.068 \\
\hline fosfomycin & 0 & 96.7 & 2.5 & 94.3 & 1.5 & 94.0 & - & 0.084 \\
\hline polymyxin B & 37.4 & 62.6 & 43.0 & 57.0 & 36.2 & 63.8 & $\uparrow$ & 0.000 \\
\hline tigecycline & 0 & 98.9 & 0 & 96.2 & 0 & 99.6 & $\downarrow$ & 0.000 \\
\hline \multicolumn{9}{|l|}{ Klebsiella spp } \\
\hline MDR & 11.9 & & 14.9 & & 11.5 & & $\downarrow$ & 0.000 \\
\hline Piperacillin/tazobactam & 15.3 & 76.3 & 21.6 & 63.5 & 20.0 & 74.6 & - & 0.266 \\
\hline Cefoperazone/Sulbactamª & 16.9 & 81.4 & 18.9 & 73.0 & 11.5 & 83.8 & - & 0.033 \\
\hline Ceftazidime/avibactam & - & - & - & - & 4.6 & 95.4 & - & NA \\
\hline Cefazolin & 41.9 & 38.7 & 40.6 & 37.5 & 30.5 & 42.4 & $\uparrow$ & 0.000 \\
\hline Cefuroxime & 22.6 & 74.2 & 40.5 & 55.4 & 18.6 & 78.0 & $\downarrow$ & 0.000 \\
\hline Ceftazidime & 22.0 & 72.9 & 37.8 & 62.2 & 24.6 & 73.1 & - & 0.445 \\
\hline Ceftriaxone & 32.2 & 66.1 & 40.5 & 56.8 & 33.8 & 63.8 & $\downarrow$ & 0.000 \\
\hline Cefepime & 10.2 & 88.1 & 20.3 & 77.0 & 9.2 & 85.4 & $\downarrow$ & 0.019 \\
\hline Cefoxitin & 16.1 & 77.4 & 21.9 & 68.8 & 11.9 & 84.7 & $\downarrow$ & 0.000 \\
\hline Moxalactam & 8.5 & 89.8 & 9.5 & 83.8 & 7.8 & 88.4 & - & 0.052 \\
\hline Aztreonam & 22.0 & 74.6 & 31.1 & 66.2 & 25.4 & 73.1 & $\downarrow$ & 0.000 \\
\hline Ertapenem & - & - & 10.8 & 82.4 & 5.4 & 93.1 & $\downarrow$ & 0.046 \\
\hline Imipenem & 10.2 & 93.2 & 9.5 & 82.4 & 4.6 & 93.1 & $\uparrow$ & 0.004 \\
\hline Meropenem & 5.1 & 96.6 & 10.8 & 87.8 & 3.8 & 96.2 & $\uparrow$ & 0.000 \\
\hline Amikacin & 3.4 & 91.5 & 1.4 & 98.6 & 0.8 & 99.2 & $\downarrow$ & 0.000 \\
\hline Gentamicin & 6.8 & 66.1 & 12.2 & 87.8 & 4.6 & 93.8 & $\uparrow$ & 0.000 \\
\hline Ciprofloxacin & 28.8 & 76.3 & 25.7 & 68.9 & 17.7 & 81.5 & $\uparrow$ & 0.000 \\
\hline Levofloxacin & 13.6 & 91.5 & 24.3 & 70.3 & 12.3 & 84.6 & $\uparrow$ & 0.000 \\
\hline Trimethoprim/Sulfamethoxazole & 8.5 & 100 & 10.8 & 89.2 & 16.2 & 83.8 & $\downarrow$ & 0.000 \\
\hline Fosfomycin & 0 & 84.7 & 5.4 & 89.2 & 3.1 & 95.4 & $\uparrow$ & 0.000 \\
\hline Polymyxin B & 15.3 & 100 & 13.5 & 86.5 & 0.8 & 99.2 & $\uparrow$ & 0.000 \\
\hline Tigecycline & 0 & 96.2 & 0 & 98.6 & 0 & 99.2 & $\downarrow$ & 0.000 \\
\hline \multicolumn{9}{|l|}{ Salmonella spp } \\
\hline MDR & 63.0 & - & 35.7 & - & 52.1 & - & $\uparrow$ & 0.042 \\
\hline Amoxicillin & 74.1 & 25.9 & 53.6 & 41.7 & 65.8 & 34.2 & $\uparrow$ & 0.000 \\
\hline
\end{tabular}


Table 2 (continued)

\begin{tabular}{|c|c|c|c|c|c|c|c|c|}
\hline & \multicolumn{2}{|c|}{2017} & \multicolumn{2}{|l|}{2018} & \multicolumn{2}{|c|}{2019} & \multirow[t]{2}{*}{ Trend } & \multirow[t]{2}{*}{$p$} \\
\hline & $\% R$ & $\% \mathrm{~S}$ & $\% R$ & $\% \mathrm{~S}$ & $\% R$ & $\% \mathrm{~S}$ & & \\
\hline Amoxicillin/Clavulanic Acid & 9.3 & 38.9 & 8.1 & 61.9 & 6.0 & 47.9 & - & 0.943 \\
\hline Piperacillin/Tazobactam & 0 & 98.1 & 2.4 & 88.1 & 0.9 & 99.1 & - & 0.128 \\
\hline Cefoperazone/Sulbactam ${ }^{a}$ & 1.9 & 96.3 & 4.8 & 92.9 & 0 & 98.3 & $\downarrow$ & 0.000 \\
\hline Ceftazidime/Avibactam & - & - & - & & 0 & 100 & - & NA \\
\hline Ceftazidime & 18.5 & 81.5 & 9.5 & 90.5 & 8.5 & 91.5 & - & 0.904 \\
\hline Ceftriaxone & 22.2 & 77.8 & 10.7 & 89.3 & 8.5 & 91.5 & - & 0.833 \\
\hline Cefepime & 13.0 & 77.8 & 4.8 & 92.9 & 5.1 & 94.0 & $\uparrow$ & 0.000 \\
\hline Moxalactam & 1.9 & 87.0 & 2.4 & 96.4 & 0 & 99.1 & - & 0.079 \\
\hline Aztreonam & 0 & 68.5 & 9.5 & 89.3 & 8.5 & 91.5 & - & 0.916 \\
\hline Ertapenem & - & - & 1.2 & 98.8 & 0 & 100 & - & 0.156 \\
\hline Imipenem & 0 & 98.1 & 1.2 & 98.8 & 0 & 100 & - & 0.059 \\
\hline Meropenem & 1.0 & 87 & 1.2 & 98.8 & 0 & 100 & $\downarrow$ & 0.008 \\
\hline Amikacin & 1.9 & 98.1 & 1.2 & 97.6 & 0.9 & 99.1 & - & 0.192 \\
\hline Gentamicin & 7.4 & 87.0 & 4.8 & 95.2 & 10.3 & 89.7 & - & 0.236 \\
\hline Ciprofloxacin & 22.2 & 22.2 & 19.0 & 31.0 & 12.0 & 35.0 & - & 0.193 \\
\hline Levofloxacin & 3.7 & 61.1 & 8.3 & 23.8 & 15.4 & 28.2 & $\uparrow$ & 0.000 \\
\hline Trimethoprim/Sulfamethoxazole & 20.4 & 79.6 & 14.3 & 85.7 & 15.4 & 84.6 & - & 0.091 \\
\hline Fosfomycin & 0 & 100 & 1.2 & 98.8 & 0.9 & 98.3 & $\uparrow$ & 0.002 \\
\hline Polymyxin B & 55.6 & 44.4 & 36.9 & 63.1 & 53.8 & 46.2 & $\uparrow$ & 0.000 \\
\hline Tigecycline & 7.4 & 92.6 & 0 & 100 & 0 & 100 & - & 0.813 \\
\hline \multicolumn{9}{|l|}{ Serratia spp } \\
\hline MDR & 12.8 & - & 5.8 & - & 5.9 & - & $\downarrow$ & 0.016 \\
\hline Piperacillin/Tazobactam & 10.3 & 85.1 & 5.8 & 90.7 & 3.6 & 91.7 & $\downarrow$ & 0.000 \\
\hline Cefoperazone/Sulbactamª & 12.8 & 82.1 & 32.6 & 64.0 & 8.4 & 88.0 & - & 0.103 \\
\hline Ceftazidime/Avibactam & - & - & - & - & 0 & 100 & - & NA \\
\hline Ceftazidime & 10.3 & 88.1 & 1.2 & 96.5 & 15.9 & 84.1 & $\downarrow$ & 0.000 \\
\hline Ceftriaxone & 33.3 & 74.6 & 37.2 & 62.8 & 19.3 & 80.7 & $\downarrow$ & 0.003 \\
\hline Cefepime & 15.4 & 80.6 & 32.6 & 65.1 & 9.5 & 83.3 & $\downarrow$ & 0.043 \\
\hline Moxalactam & 12.8 & 94.0 & 0 & 98.8 & 3.6 & 95.2 & $\downarrow$ & 0.001 \\
\hline Aztreonam & 20.5 & 76.1 & 11.6 & 60.5 & 11.9 & 83.3 & $\downarrow$ & 0.000 \\
\hline Ertapenem & - & - & 2.3 & 96.5 & 8.4 & 91.6 & $\uparrow$ & 0.015 \\
\hline Imipenem & 17.9 & 94.0 & 2.3 & 88.4 & 8.3 & 90.5 & $\downarrow$ & 0.000 \\
\hline Meropenem & 10.3 & 94.0 & 2.3 & 97.7 & 8.3 & 91.7 & $\downarrow$ & 0.000 \\
\hline Amikacin & 5.1 & 97.0 & 0 & 100 & 1.2 & 98.8 & $\downarrow$ & 0.018 \\
\hline Gentamicin & 28.2 & 79.1 & 29.1 & 70.9 & 7.1 & 92.9 & $\downarrow$ & 0.004 \\
\hline Ciprofloxacin & 36.8 & 47.8 & 41.9 & 40.7 & 16.7 & 57.1 & - & 0.930 \\
\hline Levofloxacin & 35.9 & 70.1 & 36.0 & 52.3 & 13.1 & 82.1 & - & 0.890 \\
\hline Trimethoprim/Sulfamethoxazole & 10.3 & 88.1 & 1.2 & 98.8 & 0 & 100 & $\downarrow$ & 0.000 \\
\hline Fosfomycin & 0 & 100 & 2.3 & 97.7 & 0 & 100 & $\uparrow$ & 0.020 \\
\hline Tigecycline & 5.1 & 98.5 & 0 & 98.8 & 0 & 100 & $\downarrow$ & 0.000 \\
\hline
\end{tabular}

MDR, multidrug-resistance; ESBL-E. coli, extended-spectrum $\beta$-lactamase-producing E. coli; ESBL-K. pneumoniae, extended-spectrum $\beta$-lactamase-producing $K$. pneumoniae; CR-E. coli, carbapenem-resistant E. coli; CR-K. pneumoniae, carbapenem-resistant $K$. pneumoniae; NA, no account. $\uparrow$, resistance trend with increase. $\downarrow$, resistance trend with decrease. -, no significant change

${ }^{a}$ Criteria as published by the CLSI [8] for cefoperazone also applied to cefoperazone-sulbactam 
Table 3 The susceptibility and resistance trends of non-fermentative pathogens to antimicrobial agents

\begin{tabular}{|c|c|c|c|c|c|c|c|c|c|c|c|}
\hline & \multicolumn{5}{|c|}{ All strains (204-2019) } & \multicolumn{2}{|l|}{2014} & \multicolumn{2}{|c|}{2015} & \multicolumn{2}{|c|}{2016} \\
\hline & Number & $\mathrm{MIC}_{50}$ & $\mathrm{MIC}_{90}$ & $\% R$ & $\% \mathrm{~S}$ & $\% \mathrm{R}$ & $\% \mathrm{~S}$ & $\% \mathrm{R}$ & $\% \mathrm{~S}$ & $\% R$ & $\% \mathrm{~S}$ \\
\hline \multicolumn{12}{|l|}{ A. baumannii } \\
\hline MDR & 1062 & - & - & 70.3 & - & 63.6 & - & 67.6 & - & 74.3 & - \\
\hline piperacillin/tazobactam & 1062 & 128 & 128 & 55.9 & 29.7 & 43.2 & 53.4 & 63.7 & 34.3 & 45.9 & 27.6 \\
\hline cefoperazone/sulbactamª & 1062 & 64 & 128 & 65.6 & 31.0 & 58.0 & 39.8 & 62.7 & 34.3 & 72.0 & 26.1 \\
\hline ceftazidime & 1062 & 64 & 128 & 66.4 & 32.6 & 61.4 & 37.5 & 64.7 & 32.4 & 72.8 & 26.5 \\
\hline cefepime & 1062 & 64 & 64 & 65.3 & 31.3 & 56.8 & 40.9 & 64.7 & 33.3 & 63.8 & 28.8 \\
\hline imipenem & 1062 & 32 & 64 & 63.6 & 35.9 & 52.3 & 46.6 & 60.8 & 39.2 & 71.2 & 28.8 \\
\hline meropenem & 1062 & 32 & 64 & 63.6 & 35.5 & 54.5 & 45.5 & 59.8 & 38.2 & 69.6 & 28.8 \\
\hline amikacin & 1062 & 64 & 128 & 51.5 & 46.4 & 46.6 & 51.1 & 50.0 & 50.0 & 53.3 & 44.7 \\
\hline gentamicin & 1062 & 32 & 128 & 60.4 & 35.6 & 56.8 & 38.6 & 61.8 & 38.2 & 61.9 & 35.8 \\
\hline ciprofloxacin & 1062 & 32 & 64 & 65.2 & 33.9 & 56.8 & 43.2 & 63.7 & 36.3 & 69.6 & 30.0 \\
\hline levofloxacin & 1062 & 8 & 32 & 58.6 & 35.1 & 54.5 & 40.9 & 50.0 & 37.3 & 63.4 & 30.4 \\
\hline trimethoprim/sulfamethoxazole & 1062 & 4 & 8 & 53.8 & 46.2 & 46.6 & 53.4 & 59.8 & 40.2 & 61.1 & 38.9 \\
\hline polymyxin B & 1062 & 1 & 2 & 6.7 & 93.3 & 1.1 & 98.9 & 2.0 & 98 & 12.8 & 87.2 \\
\hline tigecycline & 1062 & 1 & 4 & 4.3 & 86.5 & 4.5 & 92 & 4.9 & 90.2 & 6.6 & 81.7 \\
\hline \multicolumn{12}{|l|}{ P. aeruginosa } \\
\hline MDR & - & - & - & 21.1 & - & 22 & - & 22.8 & - & 21.2 & - \\
\hline piperacillin/tazobactam & 1044 & 2 & 128 & 10.4 & 84.5 & 8.5 & 81.7 & 5.9 & 89.1 & 9.3 & 84.5 \\
\hline cefoperazone/sulbactam ${ }^{\mathrm{a}}$ & 1044 & 8 & 64 & 12.4 & 79.9 & 13.4 & 73.2 & 6.9 & 84.2 & 11.9 & 78.8 \\
\hline ceftazidime/avibactam & 362 & 4 & 8 & 2.8 & 97.2 & - & & - & & - & \\
\hline ceftazidime & 1044 & 4 & 32 & 10.2 & 85.7 & 9.8 & 85.4 & 6.9 & 89.1 & 7.3 & 88.1 \\
\hline cefepime & 1044 & 2 & 16 & 7.8 & 88.7 & 11.0 & 85.4 & 3.0 & 94.1 & 5.2 & 90.7 \\
\hline aztreonam & 1044 & 4 & 32 & 14.4 & 76.9 & 12.2 & 78.0 & 12.9 & 78.2 & 10.4 & 79.8 \\
\hline imipenem & 1044 & 2 & 32 & 18.8 & 79.9 & 13.5 & 70.7 & 12.9 & 57.4 & 18.7 & 80.3 \\
\hline meropenem & 1044 & 0.25 & 16 & 13.5 & 83.2 & 6.1 & 92.7 & 9.9 & 86.1 & 10.9 & 86.5 \\
\hline amikacin & 1044 & 2 & 8 & 2.2 & 97.2 & 3.7 & 96.3 & 3.0 & 95.0 & 3.1 & 96.4 \\
\hline gentamicin & 1044 & 2 & 8 & 5.7 & 89.2 & 8.5 & 89.0 & 8.9 & 89.1 & 4.7 & 91.2 \\
\hline ciprofloxacin & 1044 & 0.25 & 8 & 18.2 & 75.9 & 12.2 & 82.9 & 9.9 & 86.1 & 13.5 & 84.5 \\
\hline levofloxacin & 1044 & 0.5 & 8 & 17.2 & 72.0 & 18.3 & 76.8 & 7.9 & 84.2 & 11.9 & 79.3 \\
\hline \multirow[t]{3}{*}{ polymyxin B } & 1044 & 1 & 2 & 0 & 96.5 & 0 & 100 & 0 & 93.4 & 0 & 100 \\
\hline & \multicolumn{2}{|l|}{2017} & \multicolumn{3}{|c|}{2018} & \multicolumn{3}{|c|}{2019} & \multirow[t]{2}{*}{ Trend } & \multirow{2}{*}{\multicolumn{2}{|c|}{$p$}} \\
\hline & $\% R$ & $\% \mathrm{~S}$ & & & $\% \mathrm{~S}$ & $\% R$ & & $\% \mathrm{~S}$ & & & \\
\hline \multicolumn{12}{|l|}{ A. baumannii } \\
\hline MDR & 70.4 & - & \multicolumn{2}{|c|}{72.6} & - & \multicolumn{2}{|l|}{68.2} & - & - & \multicolumn{2}{|r|}{0.841} \\
\hline piperacillin/tazobactam & 52.2 & 35.7 & \multicolumn{2}{|c|}{73.6} & 26.0 & \multicolumn{2}{|l|}{54.8} & 22.9 & $\uparrow$ & & 0.005 \\
\hline cefoperazone/sulbactam ${ }^{\mathrm{a}}$ & 66.1 & 33.9 & & & 28.4 & 61.0 & & 32.2 & - & & 0.664 \\
\hline ceftazidime & 65.2 & 33.9 & & & 32.7 & 62.7 & & 36.0 & - & & 0.438 \\
\hline cefepime & 63.5 & 33.0 & & & 28.8 & 67.1 & & 30.8 & - & & 0.062 \\
\hline imipenem & 63.5 & 34.8 & & & 33.2 & 58.9 & & 40.1 & - & & 0.858 \\
\hline meropenem & 65.2 & 34.8 & & & 32.2 & 59.2 & & 40.1 & - & & 0.920 \\
\hline amikacin & 60.0 & 29.6 & & & 46.2 & 47.6 & & 52.1 & - & & 0.749 \\
\hline gentamicin & 71.3 & 19.1 & & & 32.7 & 54.1 & & 42.1 & - & & 0.242 \\
\hline ciprofloxacin & 67.8 & 29.6 & & & 28.8 & 59.9 & & 39.0 & - & & 0.763 \\
\hline levofloxacin & 53.9 & 33.9 & & & 29.8 & 58.6 & & 41.1 & - & & 0.428 \\
\hline trimethoprim/sulfamethoxazole & 50.4 & 49.6 & & & 43.3 & 46.6 & & 53.4 & - & & 0.070 \\
\hline polymyxin B & 18.3 & 81.7 & & & 95.2 & 1.4 & & 98.6 & - & & 0.046 \\
\hline tigecycline & 2.6 & 93 & & & 88.5 & 2.1 & & 83.9 & - & & 0.081 \\
\hline
\end{tabular}


Table 3 (continued)

\begin{tabular}{|c|c|c|c|c|c|c|c|c|}
\hline & \multicolumn{2}{|c|}{2017} & \multicolumn{2}{|c|}{2018} & \multicolumn{2}{|c|}{2019} & \multirow[t]{2}{*}{ Trend } & \multirow[t]{2}{*}{$p$} \\
\hline & $\% R$ & $\% \mathrm{~S}$ & $\% R$ & $\% \mathrm{~S}$ & $\% R$ & $\% \mathrm{~S}$ & & \\
\hline \multicolumn{9}{|l|}{ P. aeruginosa } \\
\hline MDR & 18 & - & 22.1 & - & 21.8 & - & - & 0.488 \\
\hline piperacillin/tazobactam & 8.2 & 84.4 & 11.4 & 82.6 & 13.0 & 84.8 & - & 0.028 \\
\hline cefoperazone/sulbactam ${ }^{a}$ & 10.7 & 81.1 & 14.1 & 82.1 & 13.5 & 79.3 & - & 0.236 \\
\hline ceftazidime/avibactam & - & & - & & 2.8 & 97.2 & - & NA \\
\hline ceftazidime & 9.0 & 86.1 & 12.5 & 85.3 & 11.9 & 83.7 & - & 0.066 \\
\hline cefepime & 9.0 & 88.5 & 8.7 & 88.0 & 8.8 & 87.3 & - & 0.258 \\
\hline aztreonam & 14.8 & 78.7 & 15.8 & 73.9 & 16.6 & 75.7 & - & 0.067 \\
\hline imipenem & 16.4 & 82.8 & 22.8 & 37.5 & 19.1 & 79.8 & - & 0.345 \\
\hline meropenem & 12.3 & 86.1 & 15.8 & 78.8 & 16.9 & 79.8 & $\uparrow$ & 0.001 \\
\hline amikacin & 4.9 & 94.3 & 1.6 & 97.8 & 0.6 & 99.2 & - & 0.012 \\
\hline gentamicin & 8.2 & 86.9 & 6.5 & 90.8 & 3.3 & 88.1 & - & 0.029 \\
\hline ciprofloxacin & 9.8 & 89.3 & 20.1 & 69.0 & 26.2 & 65.7 & $\uparrow$ & 0.000 \\
\hline levofloxacin & 11.5 & 82.0 & 21.7 & 57.6 & 22.1 & 67.7 & $\uparrow$ & 0.001 \\
\hline polymyxin B & 0 & 100 & 0 & 93.7 & 0 & 99.7 & - & NA \\
\hline
\end{tabular}

MDR, multidrug-resistance; NA, no account. $\uparrow$, resistance trend with increase. $\downarrow$, resistance trend with decrease. -, no significant change

${ }^{\text {a }}$ Criteria as published by the CLSI [8] for cefoperazone also applied to cefoperazone-sulbactam

of the strains were resistant to polymyxin $B$ and $4.3 \%$ to tigecycline (Table 1). CR-A. baumannii (CR-AB) fluctuated between a prevalence of $55.7 \%$ and $71.2 \%$ during the surveillance time (Table 1, Fig. 2). The resistance rates in A. baumannii and $P$. aeruginosa were higher in tertiary hospitals than non-tertiary hospitals (Additional file 1: Table S2). It noted that the prevalence of CR-A. baumannii and CR-P. aeruginosa were higher in tertiary hospitals settings $(71.3 \%$ and $25.5 \%$, respectively, vs $52.0 \%$ and $12.6 \%$, respectively; both $p<0.05$ ) (Additional file 1 : Table S4). However, the prevalence of CR-A. baumannii was higher in developing areas than developed areas $(72.9 \%$ vs $56.7 \%$, respectively; $p<0.05)$ (Additional file 1 : Table S4).

Cefoperazone/sulbactam and levofloxacin were the most effective agents against Stenotrophomonas maltophilia (with $95.0 \%$ and $89.2 \%$ susceptibility, respectively) (Additional file 1: Table S5). Overall, $18.3 \%$ of S. maltophilia were resistant to trimethoprim/sulfamethoxazole. The prevalence of ceftazidime-resistant Burkholderia species was $14.3 \%$, and $24.4 \%$ of the strains were resistant to trimethoprim/sulfamethoxazole (Additional file 1: Table S5).

\section{Discussion}

During the surveillance period from 2014 to 2019, E. coli and $K$. pneumoniae were the main BSI pathogens and the proportion of MRSA and ESBL-E. coli declined, while the frequency of CR-K. pneumoniae continuously increased. We also determined that the prevalence of antimicrobial resistant pathogens, especially Gram-negative organisms (GNO), varied by hospital types and the levels of local economic development. The declining trend could be attributed to the results of a special national campaign of antimicrobial stewardship initiated in 2012 [14]. As the first national, infection-defined, surveillance program in China, the BRICS has provided more precise data to help clinicians to improve antimicrobial therapy and contain the spread of AMR.

The frequency of blood cultures is recommended for 100 to 200 blood cultures sets per 1,000 patient days [15]. The 2018 annual report of the European Antimicrobial Resistance Surveillance Network (EARS-Net) varied substantially between countries, specifying only 5.3 blood cultures per 1,000 patient days in Lithuania, compared to 206.9 in Portugal [16]. However, 100 to 200 blood cultures sets per 1,000 patient days are far from routine use in China, as the China Antimicrobial Resistance Surveillance System shows that blood culture samples account for less than $10 \%$ of total microbiological samples [17]. In China, clinical microbiology is only a branch of laboratory medicine, which is different from established clinical microbiology and infectious disease departments in other countries. As a bloodstream-infection-definition surveillance, BRICS carried out AMR studies to raise attention to blood culture and promote antimicrobial stewardship programs by providing precisive surveillance data.

The surveillance revealed that GNO constituted a major proportion of all BSI causes, which increased during the monitoring period. Furthermore, the proportion 
of $E$. coli increased rapidly, which is consistent with previous reports [18]. The SENTRY Antimicrobial Surveillance Program also revealed that detection of GNO had increased and the proportion of GNO, such as E. coli and $K$. pneumoniae, also increased [19]. The emergence of MDR GNO, such as carbapenem-resistant strains, has increased rapidly [20], which makes treatment options extremely limited in clinical practice [21] as antibiotic development is lagging behind resistance for GNO. The spillover of the resistant bacteria, which is a consequence of the fact that antibiotic-resistant bacteria can be transmitted from person to person, could be the reasons for the increasing proportion of GNO [22].

During this surveillance, it is interesting to note that MRSA decreased over time, which is in line with other surveillance results [23]. Lawes et al. suggested that antibiotic stewardship and infection control strategies might have played an important part in the reduced prevalence of MRSA [24]. However, these strategies do not fully explain why pathogens other than MRSA are becoming more prevalent rather than declining over the same period. Although specific factors responsible for changes in the rates of MRSA infection remain uncertain, molecular epidemiology might provide a better understanding of MRSA population dynamics. The phenomenon of clonal replacement, whereby clones that were once widely disseminated during a certain period may become less dominant and are replaced by other epidemic clones, has been observed worldwide $[25,26]$. In a Portuguese tertiary hospital, the Brazilian (ST239-IIIA) clone was replaced by the arrival of epidemic EMRSA-15 (ST22IV) [27]. In China, the structural change in the population of MRSA was also observed when ST5-t437 replaced ST239-t030 as the predominant genotype [28].

Although the prevalence of ESBL-E. coli declined during the surveillance period, it was still at a high level, with a prevalence of more than $50 \%$. This scenario has led to subsequent increased use of carbapenems, which was associated with the emergence and spread of carbapenem-resistant bacteria, especially among $K$. pneumoniae $[29,30]$. This phenomenon was reflected in our monitoring data in which the CR-K. pneumoniae prevalence increased quickly. Other studies in China also indicated that CR-K. pneumoniae have reached higher epidemic levels in China [31, 32]. Furthermore, evidence of a particularly higher percentage of ST11 CR-K. pneumoniae [33] indicated there was clonal spread of CR-K. pneumoniae in hospitals. Our previous study also confirmed that ST11 KPC-2-producing K. pneumoniae was the common sequence types (STs) among carbapenemase-producing Enterobacteriaceae (CPE) [34]. However, sequencing was not carried out on all isolated carbapenem-resistant Enterobacteriaceae, which needs to be further studied.
Accordingly, it is necessary to implement an antimicrobial stewardship program and effective infection control to contain and mitigate the risks of nosocomial transmission and outbreaks in hospitals, such as hand-hygiene education programs, contact precautions, and use of alert codes to promptly identify patients with CR-K. pneumoniae infections [35].

The CR-A. baumannii remained around 60\% during the surveillance period, which poses a great challenge to patient treatment, and the prevalence was similar with other surveillance programs [36]. CR-A. baumannii is an emerging concern due to the associated high mortality rates [32]. The optimal antibiotic choice for CR-A. baumannii bacteremia is controversial. Some studies have suggested the use of polymyxin as the backbone of treatment $[37,38]$, Only colistin or tigecycline still presented higher susceptibility rates in this surveillance; however, the clinical efficacy of the two agents still needs to be confirmed. Hospitals should consider antimicrobial stewardship and infection control when tackling CR-A. baumannii. Indeed, Latibeaudiere et al. demonstrated that previous CR- $A$. baumannii colonization increased the risk of developing a CR-A. baumannii infection by eightfold [39]. Targeted antibacterial therapy against infection; rather than colonization and promoting hand hygiene, environmental cleaning, and contact precautions; could be valuable strategies in the control of CR-A. baumannii [40].

It is conceivable that resistance rates are higher in tertiary hospitals where patients with more critical conditions are admitted; larger scale operations, more frequent organ transplantations, and longer hospitalizations increase a patient's risk of infection, which leads to more antibiotic use and easier induction of AMR [41]. Nonetheless, the higher AMR observed in developing regions may result from a poorer healthcare infrastructure, weaker infection control implementation, and less common antimicrobial stewardship activity. All hospitals should take individual actions to contain the spread of AMR.

There are some limitations to note in this study. First, participating hospitals covered 18 of the 31 provinces in mainland China. However, it has not yet covered all provinces and the participating hospitals are only a small proportion of the total hospitals in China. Therefore, it must be noted that by increasing the number of participants, the surveillance data might become more precise. Second, the failure to distinguish the pathogens isolated from community-acquired or hospital-acquired BSI was a major limitation. In China, blood culture is mainly requested for patients with symptoms of infection during hospitalization, which means most BSI is nosocomial BSI. Third, some relevant denominators, like patient-days, 
blood culture rates and patient characteristics, were not recorded for all patients. Last, this surveillance is not a population-based surveillance, and the incidence of BSI is not available.

\section{Conclusions}

In this report of the first national BSI surveillance program in China, E. coli and K. pneumoniae were the main BSI pathogens. The proportion of MRSA and ESBL-E. coli declined, while the frequency of CR- K. pneumoniae continuously increased. The prevalence of antimicrobialresistant pathogens, especially ESBL-E. coli, CR-E. coli, ESBL-K. pneumonia, CR-K. pneumonia and CR-A. baumannii, varied by hospital types and the level of local economic development.

\section{Supplementary Information}

The online version contains supplementary material available at https://doi. org/10.1186/s13756-022-01055-5.

Additional file 1. Supplemental Table 1. Rank order of pathogens causing bloodstream infection nationwide submitted to BRICS during 2014-2019, by hospital type and region economic development. Supplemental Table 2. The resistance of major pathogensto antimicrobial agentsby hospital level and region economic development. Supplemental Table 3. The susceptibility and resistanceof MRSA and MRCNSto antimicrobial agents. Supplemental Table 4. TheMDR prevalence ofmajor pathogens to antimicrobial agents by hospital level and region economic development. Supplement Table 5. The susceptibility and resistanceof other pathogensto antimicrobial agents. Supplemental Table 6. The susceptibility and resistanceof ESBL+, ESBL-and CR-E. colito antimicrobial agent. Supplemental Table 7. The susceptibility and resistanceof ESBL+, ESBL-and CR-K. pneumoniaeto antimicrobial agents.

\section{Acknowledgements}

We gratefully acknowledge the contributions of the members of BRICS for collection of the isolates tested in this study. Their names and affiliations are as follows: Hui Ding (Lishui City Central Hospital), Yongyun Liu (Affiliated Hospital of Binzhou Medical College), Haifeng Mao (the First People's Hospital of Lianyungang), Ying Huang (First Affiliated Hospital of Anhui Medical University), Zhenghai Yang (Yijishan Hospital of Wannan Medical College), Yuanyuan Dai (Anhui Provincial Hospital), Guolin Liao (Wuhan Puren Hospital), Lisha Zhu (The First People's Hospital of Jingzhou), Liping Zhang (People's Hospital of Ningxia Hui Autonomous Region), Yanhong Li (Anyang District Hospital of Henan Province), Hongyun Xu (The Second People's Hospital of Yunnan Province), Junmin Cao (Zhejiang Provincial Hospital of Traditional Chinese Medicine), Baohua Zhang (People's Hospital of Huangshan City), Liang Guo (Mindong Hospital of Ningde City), Haixin Dong (The Affiliated Hospital of Jining Medical University), Shuyan Hu (People's Hospital of Qingyang), Sijin Man (Tengzhou Centre People's Hospital), Lu Wang (Lu'an People's Hospital), Zhixiang Liao (Xinjiang Uygur Autonomous Region Youyi Hospital), Rong Xu (People's Hospital of Yichun City), Dan Liu (Jiujiang First People's Hospital), Yan Jin (Shandong Provincial Hospital), Yizheng Zhou (Jingzhou Central Hospital), Yiqun Liao (the First Affiliated Hospital of Gannan Medical University), Fenghong Chen (The First Hospital of Putian City), Beiqing Gu (People's Hospital of Haining City), Jiliang Wang (Shengli Oilfield Central Hospital), Jinhua Liang (The Affiliated Hongqi Hospital of Mudanjiang Medicine College), Lin Zheng (The Affiliated Hospital of Ningbo Medical School), Aiyun Li (Women's Hospital, Zhejiang University School of Medicine), Jilu Shen (The Fourth Affiliated Hospital of Anhui Medical University), Yinqiao Dong (Tianchang City People's Hospital), Lixia Zhang (Shanxi Provincial People's Hospital), Hongxia Hu (The First Affiliated Hospital of Henan University of Science and Technology), Bo Quan (The Second People's Hospital of Jingzhou), Wencheng Zhu (Lu'an Civily
Hospital), Kunpeng Liang (the Second Affiliated Hospital of Bengbu Medicine College), Qiang Liu (Huaihe Hospital of Henan University), Shifu Wang (Qilu Children's Hospital of Shandong University), Xiaoping Yan (Zigong Third People's Hospital), Jiangbang Kang (the Second Hospital of Shanxi Medical University), Xiusan Xia (the People's Hospital of Lujiang), Lan Ma (the First People's Hospital of Jiayuguan), Li Sun (The Third Hospital of Hefei), Liang Luan (General Hospital of Northern Theater Command), Jianzhong Wang (Xingang Hospital of Xinyu), Zhuo Li (The First Affiliated Hospital of Xi'an Medical University), Dengyan Qiao (Gansu Provincial Hospital of Traditional Chinese Medicine), Lin Zhang (First People's Hospital of Chenzhou), Chuandan Wan (Changshu Medicine Examination Institute), Xiaoyan Qi (Women and Children's Hospital of Jin'an District), Fei Du (Hubin Hospital of Hefei).

\section{Authors' contributions}

YC analyzed the data and drafted the paper; JJ, CY and ZL performed the experiments. QY and HK revised the analyzed data. YX designed and revised the paper. All authors contributed and approved the final manuscript.

\section{Funding}

This study was supported by Key Research and Development Program of Zhejiang province (2021 C03068) and National Nature Science Foundation of China (No. 81971984).

\section{Availability of data and materials}

Not applicable.

\section{Declarations}

Ethics approval and consent to participate

Not applicable.

\section{Consent for publication \\ Not applicable.}

\section{Competing interests}

The authors declare that they have no competing interests.

\section{Author details}

${ }^{1}$ State Key Laboratory for Diagnosis and Treatment of Infectious Diseases, The First Affiliated Hospital, Zhejiang University School of Medicine, Hangzhou 310003, China. ${ }^{2}$ Collaborative Innovation Center for Diagnosis and Treatment of Infectious Diseases, Hangzhou, China. ${ }^{3}$ Lishui City Central Hospital, Lishui, China. ${ }^{4}$ Affiliated Hospital of Binzhou Medical College, Binzhou, China. ${ }^{5}$ The First People's Hospital of Lianyungang, Lianyungang, China. ${ }^{6}$ First Affiliated Hospital of Anhui Medical University, Hefei, China. ${ }^{7}$ Yijishan Hospital of Wannan Medical College, Wuhu city, China. ${ }^{8}$ Anhui Provincial Hospital, Hefei, China. ${ }^{9}$ Wuhan Puren Hospital, Wuhuan, China. ${ }^{10}$ The First People's Hospital of Jingzhou, Jingzhou, China. ${ }^{11}$ People's Hospital of Ningxia Hui Autonomous Region, Yinchuan, China. ${ }^{12}$ Anyang District Hospital of Henan Province, Henan, China. ${ }^{13}$ The Second People's Hospital of Yunnan Province, Kunming, China. ${ }^{14}$ Zhejiang Provincial Hospital of Traditional Chinese Medicine, Hangzhou, China. ${ }^{15}$ People's Hospital of Huangshan City, Huangshan, China. ${ }^{16}$ Mindong Hospital of Ningde City, Ningde, China. ${ }^{17}$ The Affiliated Hospital of Jining Medical University, Jining, China. ${ }^{18}$ People's Hospital of Qingyang, Qingyang, China. ${ }^{19}$ Tengzhou Centre People's Hospital, Tengzhou, China. ${ }^{20}$ Lu'an People's Hospital, Hefei, China. ${ }^{21}$ Xinjiang Uygur Autonomous Region Youyi Hospital, Ürümqi, China. ${ }^{22}$ People's Hospital of Yichun City, Yichun, China. ${ }^{23}$ Jiujiang First People's Hospital, Jiujiang, China. ${ }^{24}$ Shandong Provincial Hospital, Shandong, China. ${ }^{25}$ Jingzhou Central Hospital, Jingzhou, China. ${ }^{26}$ The First Affiliated Hospital of Gannan Medical University, Ganzhou, China. ${ }^{27}$ The First Hospital of Putian City, Putian, China. ${ }^{28}$ People's Hospital of Haining City, Haining, China. ${ }^{29}$ Shengli Oilfield Central Hospital, Dongying, China. ${ }^{30}$ The Affiliated Hongqi Hospital of Mudanjiang Medicine College, Mudanjiang, China. ${ }^{31}$ The Affiliated Hospital of Ningbo Medical School, Ningbo, China. ${ }^{32}$ Women's Hospital, Zhejiang University School of Medicine, Hangzhou, China. ${ }^{33}$ The Fourth Affiliated Hospital of Anhui Medical University, Hefei, China. ${ }^{34}$ Tianchang City People's Hospital, Tianchang, China. ${ }^{35}$ Shanxi Provincial People's Hospital, Shanxi, China. ${ }^{36}$ The First Affiliated Hospital of Henan University of Science and Technology, Luoyang, China. ${ }^{37}$ The Second People's Hospital of Jingzhou, Honghu, China. ${ }^{38}$ Lu'an Civily Hospital, Beijing, China. ${ }^{39}$ The Second Affiliated Hospital 
of Bengbu Medicine College, Bengbu, China. ${ }^{40}$ Huaihe Hospital of Henan University, Kaifeng, China. ${ }^{41}$ Qilu Children's Hospital of Shandong University, Jinan, China. ${ }^{42}$ Zigong Third People's Hospital, Zigong, China. ${ }^{43}$ The Second Hospital of Shanxi Medical University, Jinzhong, China. ${ }^{44}$ The People's Hospital of Lujiang, Lujiang, China. ${ }^{45}$ The First People's Hospital of Jiayuguan, Jiayuguan, China. ${ }^{4}$ The Third Hospital of Hefei, Hefei, China. ${ }^{47}$ General Hospital of Northern Theater Command, Shenyang, China. ${ }^{48}$ Xingang Hospital of Xinyu, Xinyu, China. ${ }^{49}$ The First Affiliated Hospital of Xi'an Medical University, Xi'an, China. ${ }^{50} \mathrm{Gansu}$ Provincial Hospital of Traditional Chinese Medicine, Lanzhou, China. ${ }^{51}$ First People's Hospital of Chenzhou, Chenzhou, China. ${ }^{52}$ Changshu Medicine Examination Institute, Changshu, China. ${ }^{53}$ Women and Children's Hospital of Jin'an District, Fuzhou, China. ${ }^{54} \mathrm{Hubin}$ Hospital of Hefei, Hefei, China.

Received: 25 July 2021 Accepted: 5 January 2022

Published online: 24 January 2022

\section{References}

1. Global Action Plan on Antimicrobial Resistance: World Health Organization; [Available from: https://www.who.int/publications/i/item/ 9789241509763.

2. Lim C, Ashley EA, Hamers RL, Turner P, Kesteman T, Akech S, et al. Surveillance strategies using routine microbiology for antimicrobial resistance in low- and middle-income countries. Clin Microbiol Infect. 2021.

3. Evans RN, Pike K, Rogers CA, Reynolds R, Stoddart M, Howe R, et al. Modifiable healthcare factors affecting 28-day survival in bloodstream infection: a prospective cohort study. BMC Infect Dis. 2020;20(1):545.

4. Goto M, Al-Hasan MN. Overall burden of bloodstream infection and nosocomial bloodstream infection in North America and Europe. Clin Microbiol Infect. 2013;19(6):501-9.

5. Kern WV, Rieg S. Burden of bacterial bloodstream infection-a brief update on epidemiology and significance of multidrug-resistant pathogens. Clin Microbiol Infect. 2020;26(2):151-7.

6. Kadri SS, Lai YL, Warner S, Strich JR, Babiker A, Ricotta EE, et al. Inappropriate empirical antibiotic therapy for bloodstream infections based on discordant in-vitro susceptibilities: a retrospective cohort analysis of prevalence, predictors, and mortality risk in US hospitals. Lancet Infect Dis. 2021:21(2):241-51.

7. Zhang X, Gu B, Mei Y, Wen Y, Xia W. Increasing resistance rate to carbapenem among blood culture isolates of Klebsiella pneumoniae, Acinetobacter baumannii and Pseudomonas aeruginosa in a university-affiliated hospital in China, 2004-2011. J Antibiot (Tokyo). 2015;68(2):115-20.

8. Verboom DM, van de Groep K, Boel CHE, Haas PJA, Derde LPG, Cremer $\mathrm{OL}$, et al. The diagnostic yield of routine admission blood cultures in critically III patients. Crit Care Med. 2021;49(1):60-9.

9. Performance standards for antimicrobial susceptibility testing. 30th ed. CLSI supplement M100. Wayne, PA: Clinical and Laboratory Standards Institute; 2020

10. Hermans PE, Washington JA 2nd. Polymicrobial bacteremia. Ann Intern Med. 1970;73(3):387-92.

11. Dargère $S$, Cormier $H$, Verdon $R$. Contaminants in blood cultures: importance, implications, interpretation and prevention. Clin Microbiol Infect. 2018;24(9):964-9.

12. CDC. FAQs about choosing and implementing a CRE definition 2015 http://www.cdc.gov/hai/organisms/cre/definition.html.

13. Magiorakos AP, Srinivasan A, Carey RB, Carmeli Y, Falagas ME, Giske CG, et al. Multidrug-resistant, extensively drug-resistant and pandrugresistant bacteria: an international expert proposal for interim standard definitions for acquired resistance. Clin Microbiol Infect. 2012;18(3):268-81.

14. Xiao Y. Antimicrobial stewardship in China: systems, actions and future strategies. Clin Infect Dis. 2018;67(suppl_2):S135-s41.

15. Institute CaLS. Principles and procedures for blood cultures; approved guideline. CLSI document M47-A. 940 West Valley Road, Suite 1400, Wayne, Pennsylvania 19087-1898, USA: Clinical and Laboratory Standards Institute; 2007.

16. Surveillance of antimicrobial resistance in Europe: Annual report of the European Antimicrobial Resistance Surveillance Network (EARS-Net)
2018: The European Centre for Disease Prevention and Control; https:// www.ecdc.europa.eu/en/publications-data/surveillance-antimicrobialresistance-europe- 2018

17. China Antimicrobial Resistance Surveillance System http://www.carss. cn.

18. Laupland KB. Incidence of bloodstream infection: a review of population-based studies. Clin Microbiol Infect. 2013;19(6):492-500.

19. Diekema DJ, Hsueh PR, Mendes RE, Pfaller MA, Rolston KV, Sader HS, et al. The Microbiology of Bloodstream Infection: 20-Year Trends from the SENTRY Antimicrobial Surveillance Program. Antimicrob Agents Chemother. 2019;63(7).

20. Eichenberger EM, Thaden JT. Epidemiology and mechanisms of resistance of extensively drug resistant gram-negative bacteria. Antibiotics (Basel). 2019;8(2)

21. Xiao T, Yu W, Niu T, Huang C, Xiao Y. A retrospective, comparative analysis of risk factors and outcomes in carbapenem-susceptible and carbapenem-nonsusceptible Klebsiella pneumoniae bloodstream infections: tigecycline significantly increases the mortality. Infect Drug Resist. 2018;11:595-606.

22. Olesen SW, Lipsitch M, Grad YH. The role of "spillover" in antibiotic resistance. Proc Natl Acad Sci U S A. 2020;117(46):29063-8.

23. Klein EY, Mojica N, Jiang W, Cosgrove SE, Septimus E, Morgan DJ, et al. Trends in methicillin-resistant Staphylococcus aureus hospitalizations in the United States, 2010-2014. Clin Infect Dis. 2017;65(11):1921-3.

24. Lawes T, Lopez-Lozano JM, Nebot CA, Macartney G, Subbarao-Sharma $\mathrm{R}$, Dare $\mathrm{CR}$, et al. Effects of national antibiotic stewardship and infection control strategies on hospital-associated and communityassociated meticillin-resistant Staphylococcus aureus infections across a region of Scotland: a non-linear time-series study. Lancet Infect Dis. 2015;15(12):1438-49.

25. Albrecht N, Jatzwauk L, Slickers P, Ehricht R, Monecke S. Clonal replacement of epidemic methicillin-resistant Staphylococcus aureus strains in a German university hospital over a period of eleven years. PLoS One. 2011;6(11):e28189.

26. Cohen R, Afraimov M, Finn T, Babushkin F, Geller K, Alexander H, et al. Clonal replacement of meticillin-resistant Staphylococcus aureus during repeated outbreaks in a long-term care facility. J Hosp Infect. 2021;107:23-7.

27. Amorim ML, Faria NA, Oliveira DC, Vasconcelos C, Cabeda JC, Mendes $A C$, et al. Changes in the clonal nature and antibiotic resistance profiles of methicillin-resistant Staphylococcus aureus isolates associated with spread of the EMRSA-15 clone in a tertiary care Portuguese hospital. J Clin Microbiol. 2007;45(9):2881-8.

28. Li S, Sun S, Yang C, Chen H, Yin Y, Li H, et al. The Changing Pattern of Population Structure of Staphylococcus aureus from Bacteremia in China from 2013 to 2016: ST239-030-MRSA Replaced by ST59-t437. Front Microbiol. 2018:9:332.

29. Lowe M, Kock MM, Coetzee J, Hoosien E, Peirano G, Strydom KA, et al. Klebsiella pneumoniae ST307 with bla(OXA-181,) South Africa, 2014-2016. Emerg Infect Dis. 2019;25(4):739-47.

30. Pitout JD, Nordmann P, Poirel L. Carbapenemase-Producing Klebsiella pneumoniae, a Key Pathogen Set for Global Nosocomial Dominance. Antimicrob Agents Chemother. 2015;59(10):5873-84.

31. Hu F, Zhu D, Wang F, Wang M. Current Status and Trends of Antibacterial Resistance in China. Clin Infect Dis. 2018;67(suppl_2):S128-s34.

32. Hu F, Guo Y, Yang Y, Zheng Y, Wu S, Jiang X, et al. Resistance reported from China antimicrobial surveillance network (CHINET) in 2018. Eur J Clin Microbiol Infect Dis. 2019;38(12):2275-81.

33. Wang $Q$, Wang $X$, Wang J, Ouyang $P$, Jin C, Wang R, et al. Phenotypic and Genotypic Characterization of Carbapenem-resistant Enterobacteriaceae: Data From a Longitudinal Large-scale CRE Study in China (2012-2016). Clin Infect Dis. 2018;67(suppl_2):S196-s205.

34. Zheng B, Chen Y, Violetta L, Xiao Y, Li L. Bloodstream infections caused by Entero-bacteriaceae in China. Lancet Infect Dis. 2019;19(8):810-1.

35. Carrara E, Conti M, Meschiari M, Mussini C. The role of antimicrobial stewardship in preventing KPC-producing Klebsiella pneumoniae. J Antimicrob Chemother. 2021;76(Suppl 1):i12-8.

36. Hu FP, Guo Y, Zhu DM, Wang F, Jiang XF, Xu YC, et al. Resistance trends among clinical isolates in China reported from CHINET surveillance of bacterial resistance, 2005-2014. Clin Microbiol Infect. 2016;22(Suppl 1):S9-14. 
37. Giannella M, Bartoletti M, Gatti M, Viale P. Advances in the therapy of bacterial bloodstream infections. Clin Microbiol Infect. 2020;26(2):158-67.

38. Piperaki ET, Tzouvelekis LS, Miriagou V, Daikos GL. Carbapenem-resistant Acinetobacter baumannii: in pursuit of an effective treatment. Clin Microbiol Infect. 2019;25(8):951-7.

39. Latibeaudiere R, Rosa R, Laowansiri P, Arheart K, Namias N, Munoz-Price LS. Surveillance cultures growing carbapenem-Resistant Acinetobacter baumannii predict the development of clinical infections: a retrospective cohort study. Clin Infect Dis. 2015;60(3):415-22.

40. Munoz-Price LS, Carling P, Cleary T, Fajardo-Aquino Y, DePascale D, Jimenez A, et al. Control of a two-decade endemic situation with carbapenem-resistant Acinetobacter baumannii: electronic dissemination of a bundle of interventions. Am J Infect Control. 2014;42(5):466-71.

41. Xiao T, Zhu Y, Zhang S, Wang Y, Shen P, Zhou Y, et al. A Retrospective Analysis of Risk Factors and Outcomes of Carbapenem-Resistant Klebsiella pneumoniae Bacteremia in Nontransplant Patients. J Infect Dis. 2020;221(Suppl 2):S174-83.

\section{Publisher's Note}

Springer Nature remains neutral with regard to jurisdictional claims in published maps and institutional affiliations.

- fast, convenient online submission

- thorough peer review by experienced researchers in your field

- rapid publication on acceptance

- support for research data, including large and complex data types

- gold Open Access which fosters wider collaboration and increased citations

- maximum visibility for your research: over 100M website views per year

At BMC, research is always in progress.

Learn more biomedcentral.com/submissions 